\title{
Neural network prediction of the topside electron content over the Euro-African sector derived from Swarm-A measurements
}

\author{
Ola A. Abuelezz ${ }^{\mathrm{a}, *}$, Ayman M. Mahrous ${ }^{\mathrm{a}, \mathrm{b}}$, Pierre J. Cilliers ${ }^{\mathrm{c}}$, Rolland Fleury ${ }^{\mathrm{d}}$, \\ Mohamed Youssef $^{a}$, Mohamed Nedal ${ }^{\mathrm{e}}$, Ahmed M. Yassen ${ }^{\mathrm{a}}$ \\ ${ }^{a}$ Space Weather Monitoring Center (SWMC), Physics Dept., Faculty of Science, Helwan University, Cairo, Egypt \\ ${ }^{\mathrm{b}}$ Institute of Basic and Applied Sciences, Egypt-Japan University of Science and Technology (E-JUST), Alexandria, Egypt \\ ${ }^{\mathrm{c}}$ South African National Space Agency (SANSA), Hermanus, South Africa \\ ${ }^{\mathrm{d}}$ Lab-STICC, UMR 6285, Institut Mines-Telecom Atlantique, Campus de Brest, France \\ ${ }^{\mathrm{e}}$ Institute of Astronomy, Bulgarian Academy of Sciences, Sofia, Bulgaria
}

This study presents the first prediction results of a neural network model for the vertical total electron content of the topside ionosphere based on Swarm-A measurements. The model was trained on 5 years of Swarm-A data over the Euro-African sector spanning the period 1 January 2014 to 31 December 2018. The Swarm-A data was combined with solar and geomagnetic indices to train the NN model. The Swarm-A data of 1 January to 30 September 2019 was used to test the performance of the neural network. The data was divided into two main categories: most quiet and most disturbed days of each month. Each category was subdivided into two subcategories according to the Swarm-A trajectory i.e. whether it was ascending or descending in order to accommodate the change in local time when the satellite traverses the poles. Four pairs of neural network models were implemented, the first of each pair having one hidden layer, and the second of each pair having two hidden layers, for the following cases: 1) quiet day-ascending, 2) quiet day-descending, 3 ) disturbed day-ascending, and 4) disturbed day-descending. The topside vertical total electron content predicted by the neural network models compared well with the measurements by Swarm-A. The model that performed best was the one hidden layer model in the case of quiet days for descending trajectories, with $\mathrm{RMSE}=1.20 \mathrm{TECU}, \mathrm{R}=0.76$. The worst performance occurred during the disturbed descending trajectories where the one hidden layer model had the worst RMSE $=2.12$ TECU, $(\mathrm{R}=0.54)$, and the two hidden layer model had the worst correlation coefficient $\mathrm{R}=0.47$ ( RMSE $=1.57$ ).In all cases, the neural network models performed better than the IRI2016 model in predicting the topside total electron content. The NN models presented here is the first such attempt at comparing NN models for the topside VTEC based on Swarm-A measurements.

Keywords: Neural network; Swarm satellite; Topside vertical electron content; IRI2016 model

\footnotetext{
* Corresponding author.

E-mail addresses: ola.abuelezz@gmail.com (O.A. Abuelezz), ayman. mahrous@ejust.edu.eg (A.M. Mahrous), pjcilliers@sansa.org.za (P.J. Cilliers), rolland.fleury@imt-atlantique.fr (R. Fleury), mnedal@astro. bas.bg (M. Nedal).
}

\section{Introduction}

Observations of the topside ionosphere by the Swarm-A satellite can complement the bottom-side ionospheric measurements by ionosondes (Huang and Reinisch, 2001). The largest part of the TEC comes from the topside ionosphere (h $>\mathrm{hmF} 2$ ) with contribution estimated to be from $65 \%$ (Belahaki and Tsuagori, 2002), to 80\% (Bilitza, 2009). 
Therefore, it is important to establish a topside electron content model which may be useful for telecommunication purposes.

The ionosphere, being part of the upper atmosphere of Earth, is a highly variable environment. The ionospheric electron density varies spatially with altitude and latitude, and temporally with a time of day, season, and solar activity (Wintoft and Cander, 2000). The TEC is an important ionospheric feature that can be used for several purposes, such as the studies of the ionosphere-plasmasphere system and space weather applications, as well as for Global Navigation Satellite Systems (GNSS) applications (Stankov et al., 2010). The TEC, measured along the path of a GNSS radio signal, represents the total number of electrons in a cylinder of a $1 \mathrm{~m}^{2}$ cross-sectional area along the path of the GNSS radio signal. In general, the slant TEC (STEC) can be calculated by taking the line integral of the threedimensional electron density $\left(n_{e}\right)$ along the signal path (s) from a GNSS receiver to the GNSS satellite (Bothmer and Daglis, 2007). The vertical TEC (VTEC) at the ionospheric pierce point of the ray path is derived from the STEC by using a geometric mapping function (Liu et al., 2009).

The ionospheric variability as described by TEC variations has been analysed for magnetically the quiet and disturbed days. Negative or positive ionospheric storms during disturbed days can be characterized by either a decrease or increase of TEC and electron density with respect to quiet time behaviour (e.g., Prölss, 1993a, 1995; Fuller-Rowell et al., 1994; Buonsanto, 1999; Tsagouri et al., 2000; Matamba et al., 2015).

It is known that modelling ionospheric TEC during disturbed days is a big challenge (Fuller-Rowell et al., 2000; Habarulema, 2010, 2011; Uwamahoro, and Habarulema, 2015). Several other researchers have attempted to predict and forecast the TEC during both quiet and disturbed conditions (Mao et al., 2005, 2008; Habarulema, 2010; Ercha et al., 2012; Watthanasangmechai et al., 2012).

We have utilized the neural network (NN) technique that has been demonstrated by several authors (e.g., Habarulema and McKinnell, 2012; Habarulema, 2010; Habarulema et al., 2009; Okoh, 2016) as a very efficient tool for ionospheric modelling and forecasting. The strengths and versatility of neural NNs are derived from their ability to represent both linear and nonlinear relationships between inputs and outputs directly from the input data (Baboo and Shereef, 2010).

TEC prediction using the NN technique has been done over many years with relative success. An artificial NN has been used in ionospheric studies that applied large amounts of solar-terrestrial and ionospheric data to predict the temporal and spatial variations of the ionospheric critical frequency foF2 and TEC Cander, 1998). The Middle East Technical University Neural Network (METU-NN) model, a data-driven neural network model of one hidden layer, was used for forecasting and nowcasting of TEC values of the ionosphere during space weather events
(Tulunay et al., 2006). The first NN Global Positioning System (GPS) prediction model over South Africa based on TEC measurements was developed by Habarulema et al. (2009). The model comprised a feed forward neural network $(\mathrm{NN})$ trained with data from 10 GPS receiver stations over five years. TEC predictions over southern Africa were produced by means of the regional southern Africa Total Electron Content Prediction (SATECP) NN model based on GPS data (Habarulema et al., 2011). The application of NN modelling for forecasting the ionospheric TEC over China was demonstrated by Song, et al. (2018), using the genetic algorithm to optimize the initial weights of the NN. The first regional total electron content (TEC) model over the entire African region, the AfriTEC model, was developed by Okoh et al. (2019) using observations for years 2000 to 2017 from terrestrial GPS receivers and GPS receivers on the COSMIC satellites.

The modelling of topside ionosphere has been attempted by Coïsson et al., (2002) through a comparison of the topside electron concentration profiles of the IRI and NeQuick models with measurements by the Intercosmos-19 satellite for many different geophysical situations during a period of high solar activity (March 1979 to December 1980). They concluded that topside modelling of both the IRI and NeQuick needed improvement. Improvements in the Bent model for the topside electron density of the IRI were proposed by Depuev and Pulinets (2004) using the database of Intercosmos- 19 satellite topside soundings during a period of high solar activity. Attempts were made to accommodate longitudinal dependencies in the empirical model they proposed. The modelling of the electron density values topside ionosphere has been attempted by Pignalberi et al. (2018) using electron density recorded by Swarm satellites from December 2013 to June 2016 and foF2 and $\mathrm{hmF} 2$ values provided by IRI UP (International Reference Ionosphere UPdate). They assumed that the scaling height in the topside region was constant and demonstrated that the $\alpha$-Chapman analytical function gave the best performance. Topside total electron content (TEC) values derived from the GOCE and TerraSAR-X low earth orbit satellites were used by Ren et al., 2020) to validate the topside ionosphere predictions of the NeQuick2 and IRI-2016 models from 2008 to 2018. Their results showed that these two models both underestimate the topside ionosphere. The variation of the VTEC over Antarctica during 20112017 was studied by Tariku (2020). The pattern of variation of the VTEC inferred from the IRI 2016, IRI-Plas 2017 and NeQuick 2 models was demonstrated to be generally smaller than the GPS-derived VTEC values. From the literature, it is clear that there is a gap on modelling of the topside VTEC.

In this work, we introduce the first regional Swarm-NN model for VTEC in the topside ionosphere trained over the Euro-African sector using data over the period from January 2014 to December 2018 and tested with Swarm-A data from 1 January to 30 September 2019. Particularly, we have utilised the NN technique to describe the quiet 
and disturbed periods of VTEC obtained from Swarm-A satellite measurements through the ascending and descending trajectories. In Section 2, we describe the data and methods used in the study. In Section 3, we present the results and discussion and the comparison of the neural network model with the IRI2016 model. In Section 4, we present the conclusion of the study.

\section{Data and methodology}

Data used in this work include topside electron content values at a temporal resolution of $1 \mathrm{sec}$ that were derived from Swarm-A data, as well as solar and geomagnetic indices obtained from the OMNI database.

\subsection{Data}

\subsubsection{Swarm satellite}

Swarm is a recent mission of the European Space Agency (ESA) that was launched in November 2013 with the aim of studying the dynamics of the Earth's magnetic field and its interactions with the Earth system. It consists of a constellation of three satellites (Swarm-A, -B and -C). The three Swarm satellites fly in the topside ionosphere. Each satellite has an on-board GPS receiver of which the data can be used for deriving the topside ionospheric TEC along the ray path between the Swarm and GPS satellites (Zakharenkova, Astafyeva 2015). Swarm A and C fly at an altitude of $460 \mathrm{~km}$ with a $1.5^{\circ}$ longitudinal spacing and an inclination of $87.4^{\circ}$. Swarm B flies at an altitude of $540 \mathrm{~km}$ with an inclination $86.8^{\circ}$. During a day, the Swarm satellites complete about 15 to 17 polar orbits in an average $90 \mathrm{~min}$ per orbit. The orbital planes of Swarm-B and Swarm-A/C differ by $9 \mathrm{~h}$ of local time (Fiori et al., 2013; 2014). Swarm satellites regress in longitude around $23^{\circ}$ between orbital ascending nodes. Swarm$\mathrm{A}$ and $-\mathrm{C}$ need about 133 days to cover all $24 \mathrm{~h}$ of local time and Swarm-B needs about 141 days (Xiong et al., 2016b). The local time remains almost the same across most of the latitudes in each crossing on a particular day during the ascending or descending trajectories. Data sets measured by Swarm can be downloaded from (http://earth.esa.int/Swarm). The cadence of L2-TEC data is $1 \mathrm{~Hz}$.

One Swarm satellite can communicate simultaneously with multiple GNSS satellites; hence, there can be multiple STEC values for a given universal time (UT) (Swarm, 2013). The Swarm VTEC is derived from the mean of the Swarm STEC values (Zakharenkova and Astafyeva, 2015). The topside VTEC is here modelled and predicted as a function of physical and geophysical parameters that include season (day of year), time of day, as well as solar and geomagnetic activities.

\subsubsection{Solar and geomagnetic indices}

The solar wind data and geomagnetic indices were obtained from the OMNI database (http://omniweb. gsfc.nasa.gov/) during the descending phase of the solar cycle 24. Data from 2014 to 2018 were used for training our model, whereas data of 2019 were used for testing the performance of the model. The solar and geomagnetic inputs for the $\mathrm{NN}$ included the solar radio flux density at wavelength $10.7 \mathrm{~cm}$ (F10.7 index), and the disturbed storm time (Dst) index. The Dst index is a scale of geomagnetic activity used to express the acuteness of magnetic storms. The ten most quiet days and the five most disturbed days in each month were selected based on the Dst index by using the sequence of the degree of quietness and disturbance numbered as follows 1q, 2q, 3q, 4q, 5q, 6q, 7q, 8q, $9 \mathrm{q}$ and $0 \mathrm{q}$, and $1 \mathrm{~d}, 2 \mathrm{~d}, 3 \mathrm{~d}, 4 \mathrm{~d}$ and $5 \mathrm{~d}$, respectively (http://wdc.kugi.kyoto-u.ac.jp/qddays/format.html).

The F10.7 index is a daily measurement indicator for solar activity, given in solar flux units (s.f.u.), and varies from ( $50 \geq \mathrm{F} 10.7 \geq 300)$ through a solar cycle. To represent influence of the solar wind on TEC, the proton fluxes at the $\mathrm{L} 1$ point in three energy bands (i.e $10,30,60 \mathrm{MeV}$ ) are also used as inputs to the NN.

\subsection{Methodology}

Pre-processing of Swarm-A data involved the extraction of VTEC values from STEC between Swarm-A and GPS altitudes. The Swarm-A STEC values are along the ray paths from Swarm-A satellite to the GPS satellites. There are typically five GPS satellites in view at each epoch. The VTEC value at each $1 \mathrm{sec}$ epoch is derived from the mean of the STEC values by using a geometric mapping function for each satellite.

After that, The VTEC values are selected for elevation $>50^{\circ}$ as recommended in the Swarm Level 2 TEC product description.

1- We separated the ascending and descending trajectories of the Swarm-A passes, because they occurred at two different local times e.g. ascending and descending at 10:20 LT and 22:22 LT respectively on 201709-06 over the African sector.

2- We filtered the data by geographic longitude and latitude to cover the Euro-African region, defined by the coordinate bounds $-71.5<$ latitude $<71.5$ and $-20<$ longitude $<50$.

We inspected the correlation between potential input variables and Swarm VTEC values and used a correlation matrix to select only nine inputs. The 9 input parameters were selected from the error matrix, which demonstrates the performance of the model. The error matrix is often used in the field of machine learning for classification problems (see Stehman, 1997; Powers, 2011). It shows how much each predicted class is correlated with its corresponding drivers. The 9 input parameters were taken as the day of the year (DOY), the hours of the day rounded to the nearest integer (HR), latitude, longitude, Dst index, F10.7 index, and the proton fluxes at 10, 30, and $60 \mathrm{MeV}$. To convert the seasonal and diurnal variations into numerically continuous parameters, the DOY and 
HR are split into two cyclical components defined by (Habarulema, 2010)

$$
\begin{aligned}
& D O Y_{s}=\sin \left(\frac{2 \pi \times D O Y}{365.25}\right) D O Y_{c}=\cos \left(\frac{2 \pi \times D O Y}{365.25}\right) \\
& H R_{s}=\sin \left(\frac{2 \pi \times H R}{24}\right) H R_{c}=\cos \left(\frac{2 \pi \times H R}{24}\right)
\end{aligned}
$$

where DOYs, DOYc, HRs, and HRc are the sine and cosine components of the DOY and HR, respectively. Therefore, 11 input parameters were used in the final NN model, namely DOYs, DOYc, HRs, HRc, latitude, longitude, Dst, F10.7, PF $10 \mathrm{MeV}$, PF $30 \mathrm{MeV}$, and PF $60 \mathrm{MeV}$. Before the training of the $\mathrm{NN}$ model, the dataset was divided into two categories for training and testing purposes. Each category was spilt corresponding to the ionospheric variability (e.g. quiet and storm conditions). Furthermore, each category showed the latitude, longitude and HR dependency through separating the ascending and descending trajectories of Swarm-A.

The quietest and the most disturbed days of each month were derived from the WDC database (http://wdc.kugi.kyoto-u.ac.jp/). The time resolution of the measured topside VTEC is $1 \mathrm{~s}$ and the actual topside VTEC that obtained from Swarm-A data were extracted for a period of five years $(2014,2015,2016,2017$ and 2018) from the dataset and used in NN training process. Then data from the first 9 months of 2019 were used for testing the NN performance. This procedure is to make sure that the NN was not being over-trained but sufficiently generalized.

We tested two NN architectures for each relationship: a $\mathrm{NN}$ with a single hidden layer and NN with double hidden layers, with 20 hidden nodes each. The single hidden layer (1HL) NN refers to a NN with only one hidden layer, besides the input and output layers. The two hidden layer (2HL) NN refers to a NN with two hidden layers, besides the input and output layers.

After inspecting the correlation of the VTEC values with different potential inputs we selected only 11 input parameters that had the highest correlations with the VTEC values. The numbers of daily data sets used for training and testing for the quiet days ascending (QD Asc), quiet days descending (QD Des), disturbed days ascending (DD Asc), and disturbed days descending (DD Des) are shown in Table 1.

In a few cases, there was no Swarm data available on the disturbed days or quiet days of some months in the year.

\subsection{The neural network model}

The NN consists of a number of processing units called neurons or perceptrons linked by weighted connections between the layers (Hopfield, 1982). Each unit receives inputs from other units in the previous layer and generates a single output. The output is then forwarded as an input to all the other neurons in the subsequent layers. NNs consist of three main sections (Fig. 1); the input layer, which interfaces with the input variables, the hidden layer(s), where the learning process is performed, and the output layer which includes the target variable. The layers in a multilayer neural network are fully connected, that is, every neuron in each layer is connected to every other neuron in the subsequent layer. The $\mathrm{NN}$ is considered as an iterative learning process in which it maps the embedded relation between the inputs and the output when given enough training examples.

The NN performance strongly depends on the NN architecture and it is different from one application to another. The number of hidden layers and hidden neurons depends on the complexity of the problem under study, but can lead to overfitting in which the model is

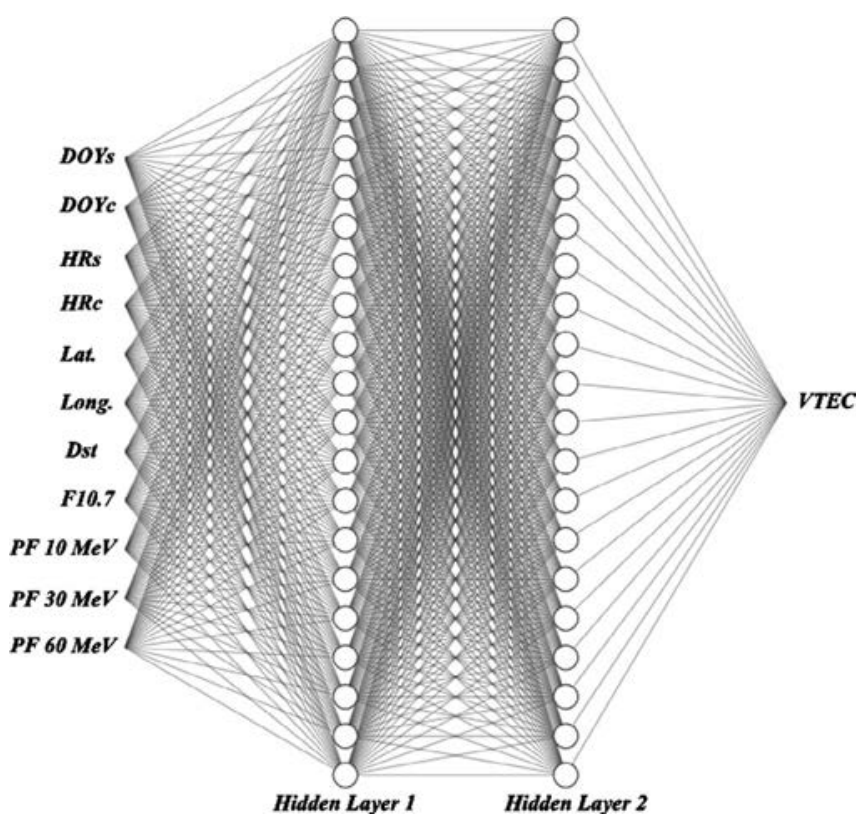

Fig. 1. Illustration of the NN architecture with labelled inputs and output.

Table 1

Number of quiet days (QD) (on average 10 days per month) and disturbed days (DD) (on average 5 days per month) for ascending (Asc) and descending (Des) passes per year for each year of Swarm-A data used.

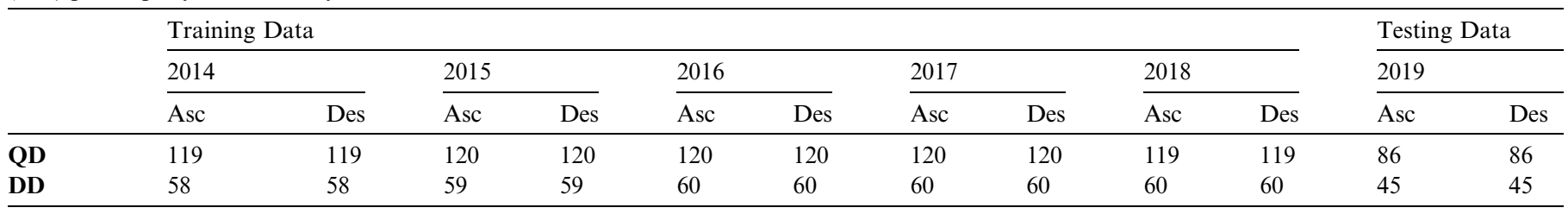


closely fit to the training data but fails to fit the testing data. So, an efficient NN model is characterized by the ability to be generalized over a wide range of data it has not encountered before. Yet, there is no simple rule that can determine the optimum number of hidden layers and hidden neurons for an application. The architecture/topology used is determined by trial and error, and the best architecture/topology in the present case was chosen as the one with the lowest RMSE over the period of interest. The output of a single-hidden layer NN can be written as

$\left.G_{k}=f \sum_{j=1}^{M} H_{j} w_{j, k}^{(2)}+b_{k}\right)$

where $G_{k}$ is the network output at the $k$-th output of $K$ output nodes, $M$ the number of hidden nodes in the first hidden layer, $w_{j, k}^{(2)}$ the weights of the links between the $j$-th hidden node and the $k$-th output node, $b_{k}$ the biases connected to the output nodes, $f$ the activation function, and $H_{j}$ the output of the $j$-th hidden node, which can be represented as
$\left.H_{j}=f \sum_{i=1}^{N} x_{i} w_{i, j}^{(1)}+b_{j}\right)$

where $x_{i}$ the input values, $N$ the number of inputs nodes, $w_{i, j}^{(1)}$ the weights of the links between the $i$-th input and the $j$-th node of the first hidden layer, and $b_{j}$ the biases connected to the first hidden layer. For a double-hidden layers $\mathrm{NN}$, the output can be written as

$\left.y_{l}=f \sum_{k=1}^{S} G_{k} w_{k, l}^{(3)}+b_{l}\right)$

where $y_{l}$ is the output of the $l$-th output node, $G_{k}$ represent the outputs of the previous hidden layer, $S$ the number of hidden nodes in the second hidden layer, $w_{k, l}^{(3)}$ the weights of the links between the k-th hidden node of the second hidden layer and the $l$-th output node, and $b_{l}$ the bias connected to the $l$-th output nodes.

We used a semi-automatic method to find the best architecture for any specific regression problem. This method involves using two nested for-loops in which the number of loops represents the number of hidden layers and each loop represents the number of hidden neurons in this speci-

\section{Training Data}

Testing Data
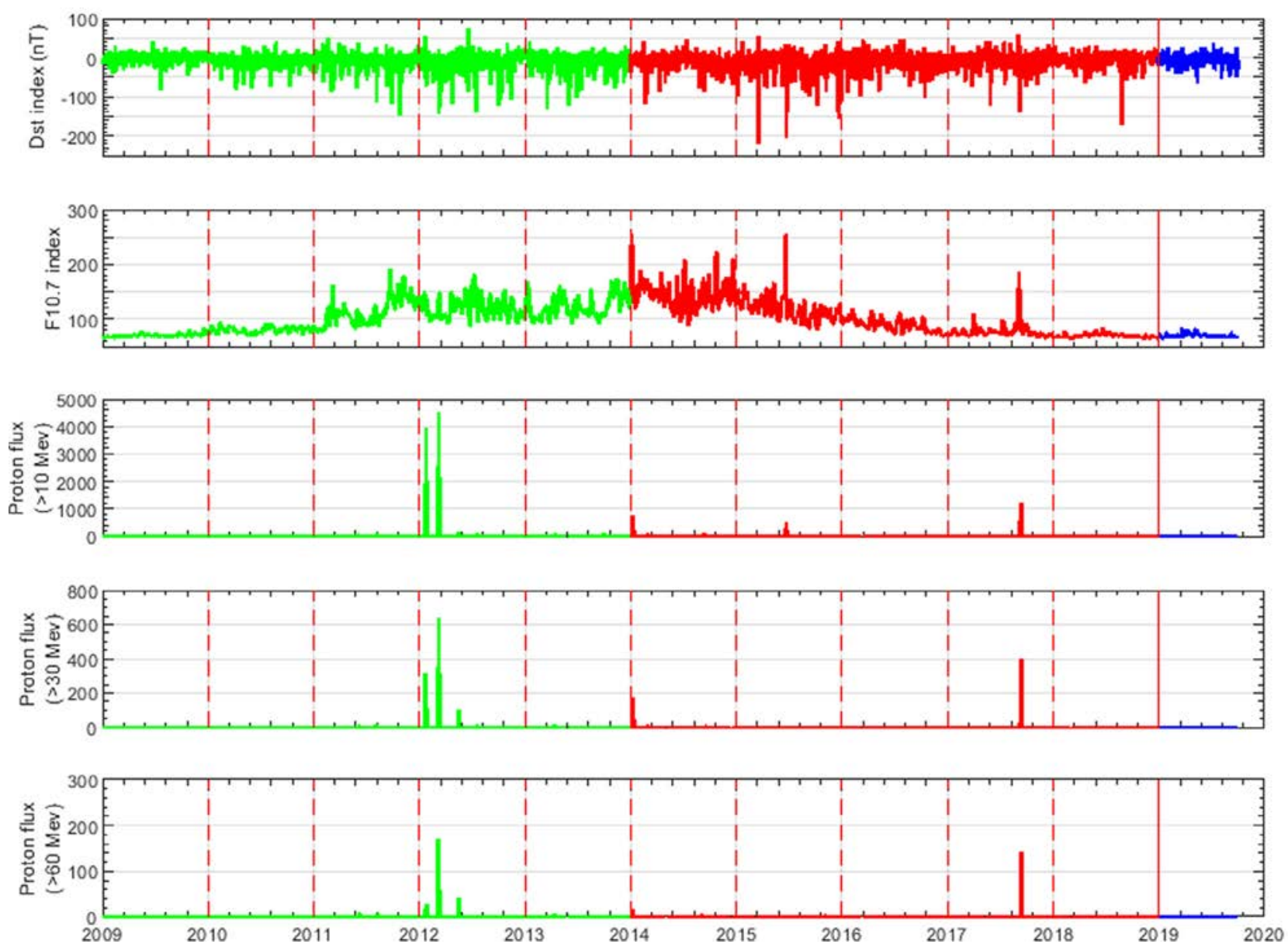

Fig. 2. The Dst index (nT) and the F10.7 index (sfu) and the solar proton fluxes at 3 energy channels $(\mathrm{PF}>10 \mathrm{MeV}, \mathrm{PF}>30 \mathrm{MeV}$, and $\mathrm{PF}>60 \mathrm{MeV}$ ) during the last 10 years. The time period spans from 1 January 2014 to 30 September 2019. All data are 1-hour-averaged data from the OMNIWeb Service. 
fic hidden layer. Each NN topology runs two consecutive times to estimate the average response of this particular NN architecture. The Root-Mean-Squared Error (RMSE) and the percentage error are recorded for each NN topology and we selected the NN topologies that gave the minimum errors.

We chose the feed-forward back-propagation $\mathrm{NN}$ as it is one of the most popular approaches of machine learning and it performs quite effectively for space weather applica- tions (see Qahwaji and Colak, 2006; Ajabshirizadeh et al., 2011; Bortnik et al., 2018). Besides, it can approximate any input-output map with concurrent inputs and outputs efficiently (see Haykin, 1999; Bakr and Negm, 2012). We used the Levenberg-Marquardt optimization (Levenberg, 1944; Marquardt, 1963) as a learning rule for our model because it performed better than other training functions for our application. The training process in the back-propagation algorithm of a neural network involves two phases, namely

(a)

QD Asc VTEC
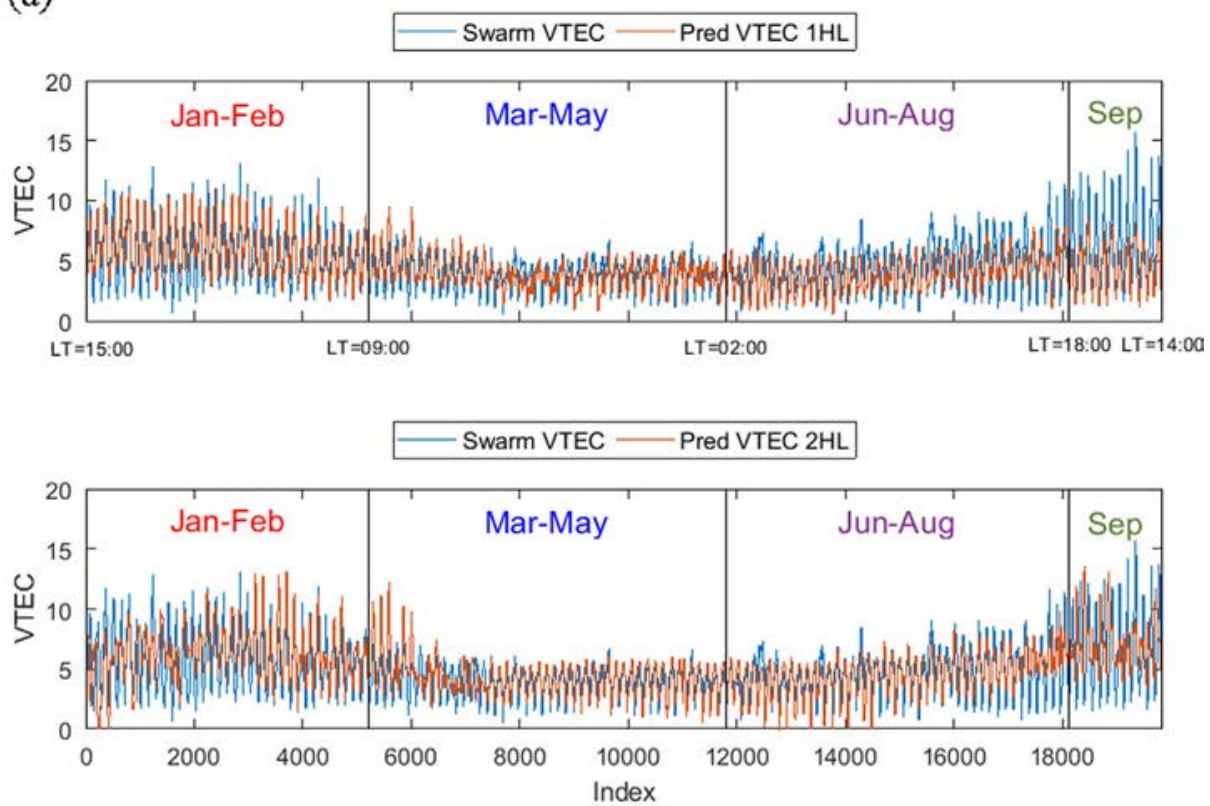

(b)
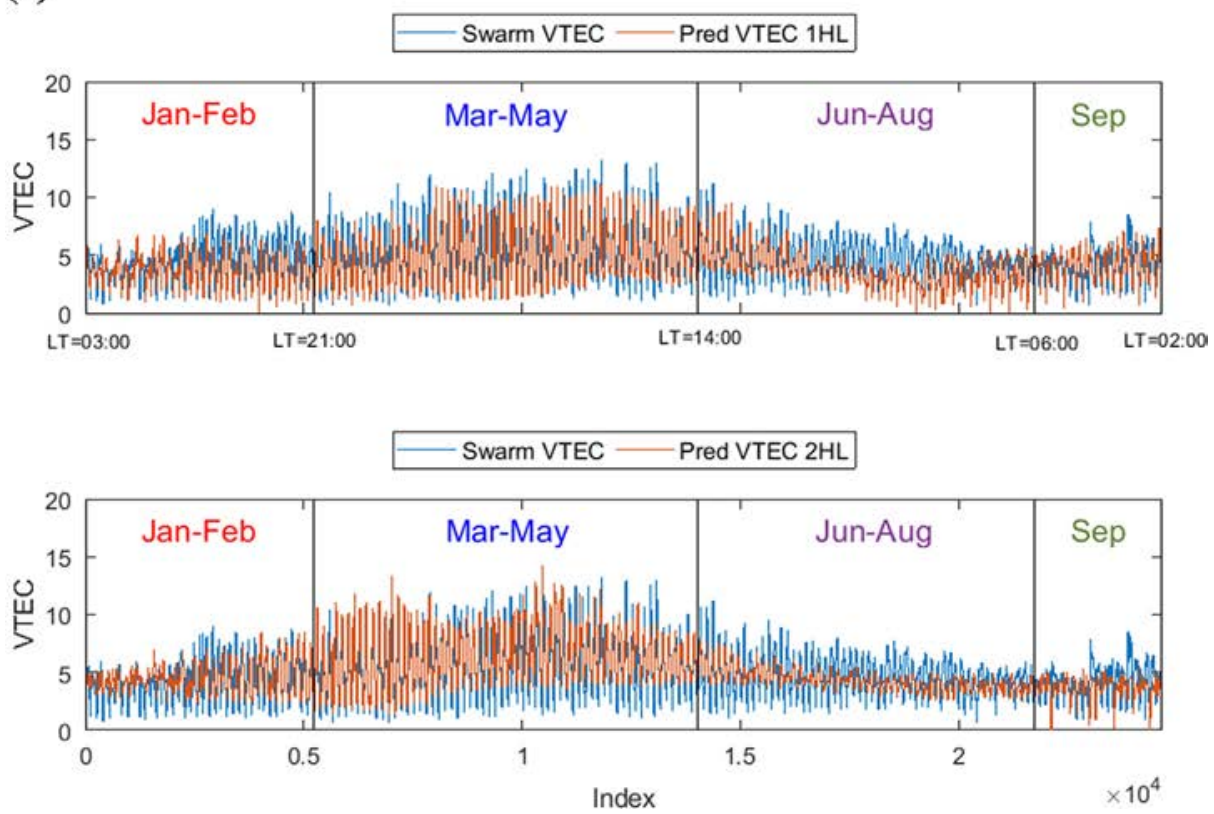

Fig. 3. (a,b). Comparison between the observed and predicted VTEC during ascending (a, QD Asc) and descending (b, QD Des) passes of Swarm-A on geomagnetically quiet days. The observed VTEC was derived from Swarm-A and the predicted VTEC was derived from NN models with one hidden layer (1HL) and two hidden layers (2HL) respectively. 


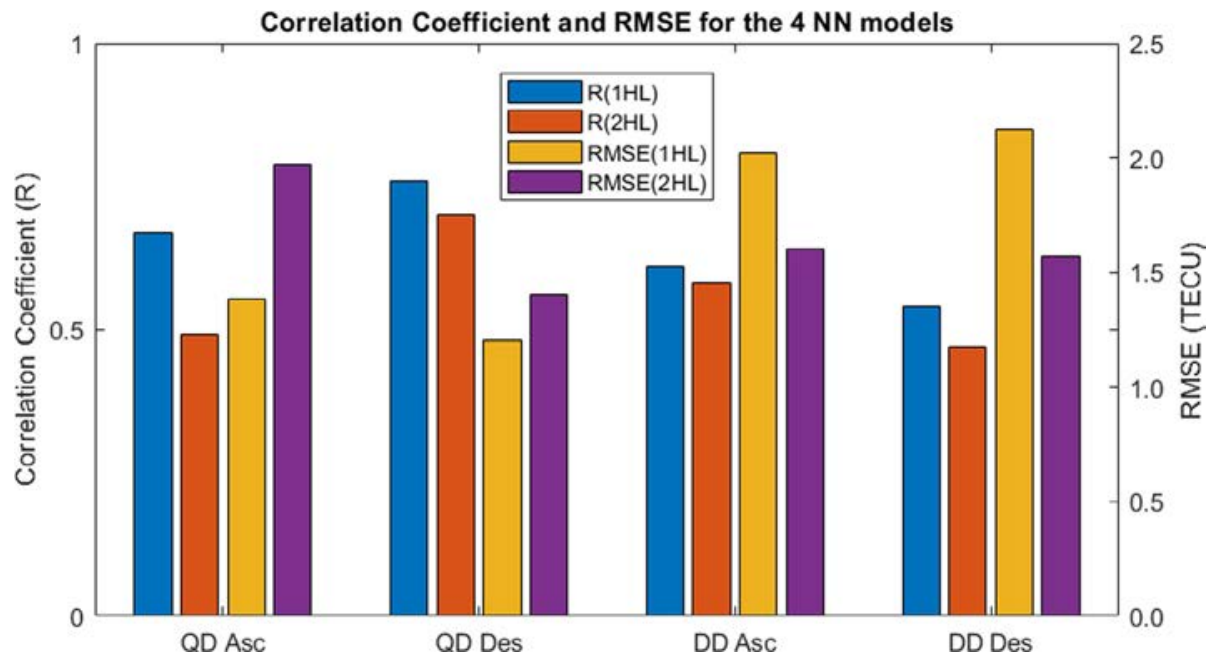

Fig. 4. The correlation coefficients and the RMSE values in TECU between measured VTEC, by means of Swarm-A, and NN VTEC for the single hidden layer (1HL) and double hidden layer (2HL) models for each of the ascending (Asc) and descending (Des) passes of Swarm-A for quiet days (QD) and disturbed days (DD) during 2019.

forward and backward. The back-propagation algorithm is assumed to converge when the error per iteration (i.e., epoch) is satisfactorily small.

To assess the performance of the NN, RMSE was computed. The RMSE is defined as
$\mathrm{RMSE}=\sqrt{\frac{\sum_{i=1}^{N}\left(V T E C_{\text {meas }}-V T E C_{\text {pred }}\right)^{2}}{N}}$,
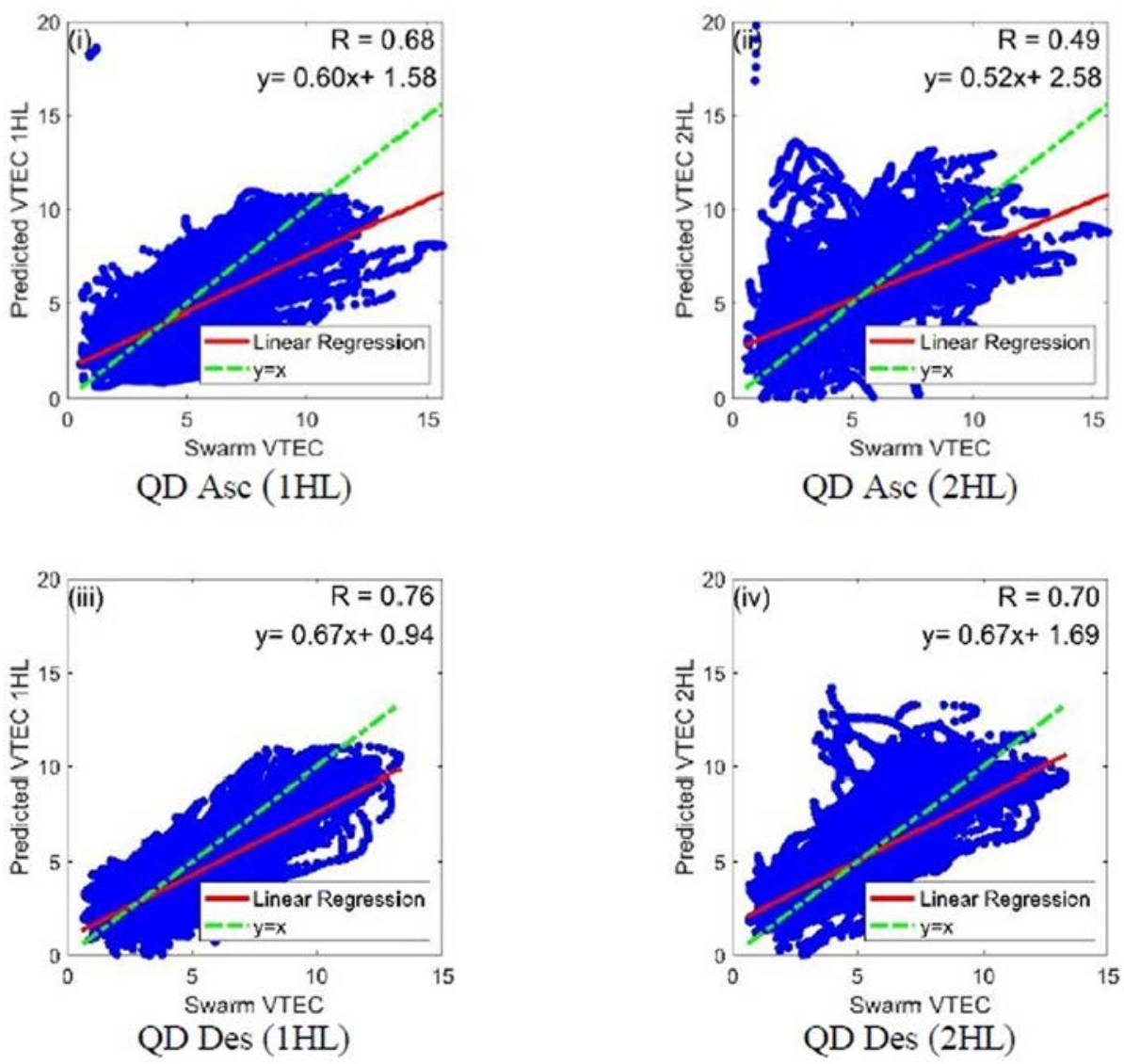

Fig. 5. Scatter plots of the observed VTEC values derived from Swarm-A data and the predicted VTEC from the NN (1HL) and NN (2HL) for the four models (QD Asc, QD Des, DD Asc and DD Des). The correlation coefficients for each case are given in the top right-hand corner of the plots. 

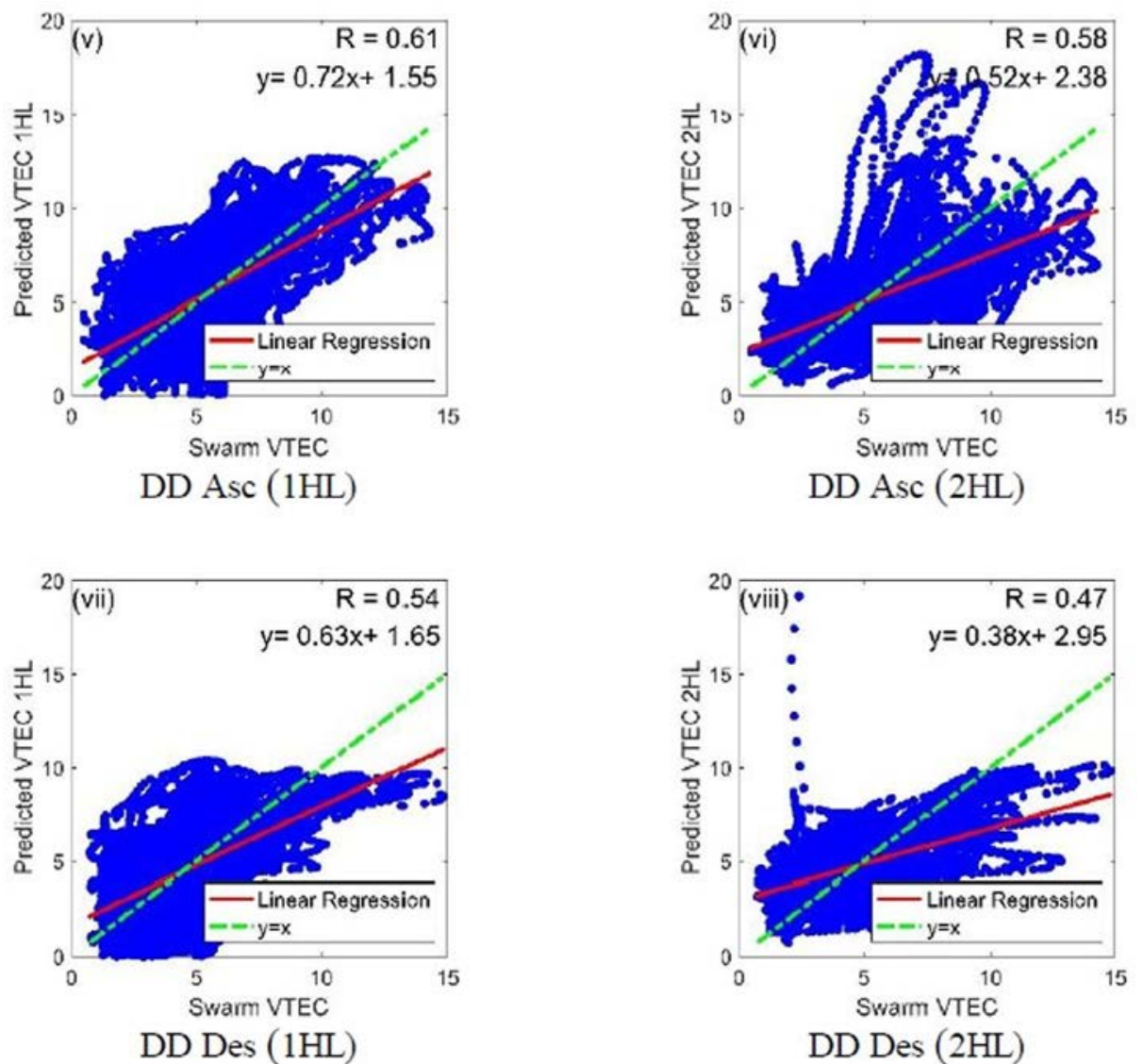

Fig 5. (continued)

where $N$ is the number of data points, VTE $C_{\text {pred }}$ is VTEC predicted by the NN and $V T E C_{\text {meas }}$ is the VTEC derived from Swarm-A measurements.

The Pearson correlation coefficient $(\mathrm{R})$ between the Swarm and NN (VTEC) as given by

$R=\frac{n(\Sigma x y)-(\Sigma x)(\Sigma y)}{\sqrt{\left[n \Sigma x^{2}-(\Sigma x)^{2}\right]\left[n \Sigma y^{2}-(\Sigma y)^{2}\right]}}$

was used as additional measure of the performance of the $\mathrm{NN}$. Here the numerator is the covariance between the variables $\mathrm{x}$ and $\mathrm{y}$ and the denominator is the product of the standard deviation of $\mathrm{x}$ and $\mathrm{y}$. The best NN architecture was taken as the one with the minimum RMSE and highest correlation coefficient over the whole period of interest for the selected dataset.

\section{Results and discussion}

This section describes the results of the NN and predicting topside VTEC values over the Euro-African region. The dataset was derived from Swarm-A measurements during the period 1 January 2014 to 30 September 2019. The time series were divided into two periods for each of four models. The data for the period $2014-2018$ were used for training the NN and from 1 January 2019 to 30 Septem- ber 2019 were used for testing the predicted topside VTEC values.

Fig. 2 shows the Dst index, the F10.7 index, and the solar proton fluxes in three energy channels for the full solar cycle from 2009 to 2019 . The green parts show the period preceding the launch of the Swarm satellites to provide the context of the data used for training the NN. The periods used for training and testing of the $\mathrm{NN}$ are shown in red and blue respectively. The highest levels of proton flux in all 3 energy channels occurred during the storm of 7 September 2017 when the Dst index reached -124 nT and F10.7 reached $185.5 \mathrm{sfu}$, namely Proton flux $(>10 \mathrm{MeV})=1208$ per $\mathrm{cm}^{2}$-sec-ster, Proton flux $(>30 \mathrm{MeV})=404$ per $\mathrm{cm}^{2}$-sec-ster and Proton flux $(>60 \mathrm{MeV})=142$ per $\mathrm{cm}^{2}$-sec-ster. The training data covered half of the solar cycle and started near the peak of the solar cycle while the testing data was taken from the end of the solar cycle. Note that while the training data included several strong storms (Dst $\leq-150 \mathrm{nT}$ ), the testing data had no strong storms (Dst $\geq-50 \mathrm{nT}$, Pf $\leq 0.36 \mathrm{MeV}$ ), Since Dst hardly went below $-50 \mathrm{nT}$ as well as very low proton flux levels. This might have contributed to the fact that the DD models had lower correlations and higher RMSE values in general compared to QD models.

Fig. 3(a) and (b) show the time-series graphs of the comparison between the measured and estimated VTEC from 
January to September 2019 for the single hidden layer NN (1HL) and the double hidden layer NN (2HL).

The measured topside VTEC is obtained from SwarmA. The x-axis in Fig. 3 represents 9 months of year 2019, which was used as the testing set. The $\mathrm{x}$-axis is labelled "Index" because it is chronological but not sequential in time. Hence, there is no direct link between the index and the day of the month. The results are labelled by the months which represent the solstices and equinoxes of the year by the following periods: "Jan-Feb" represents the solstice period 1 January to 28 February 2019), ("MarMay" represents the equinox period 1 March to 31 May 2019), ("Jun-Aug" represents the solstice period 1 June to 31 August 2019), ("Sep" represents the equinox period 1 September to 30 September 2019). We have avoided the word "season" since every pass of the Swarm satellite over the Euro-African sector traverses both northern and southern hemispheres, which have opposite seasons. In the top panel, the LT is shown at the transitions between these periods. This format of the results facilitates comparison of the overall the variations of the range of VTEC values during different part of the testing year.

The local time (LT) difference between the ascending and descending passes is about $12 \mathrm{~h}$. Note that, between two successive ascending equatorial crossings of SwarmA, the LT decreases by $1.28 \mathrm{~min}$ and the longitude decreases by about $23^{\circ}$ (Xiong et al., 2016b).

Fig. 3 shows the strong dependency of the diurnal VTEC variation on the LT. The largest variation of the topside VTEC (16 TECU) occurs between noon and dusk (i)

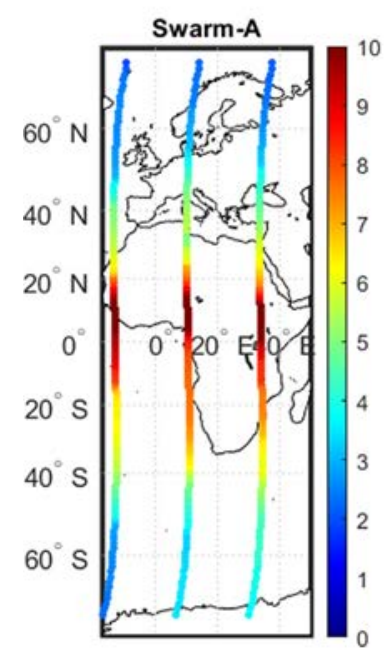

(ii)

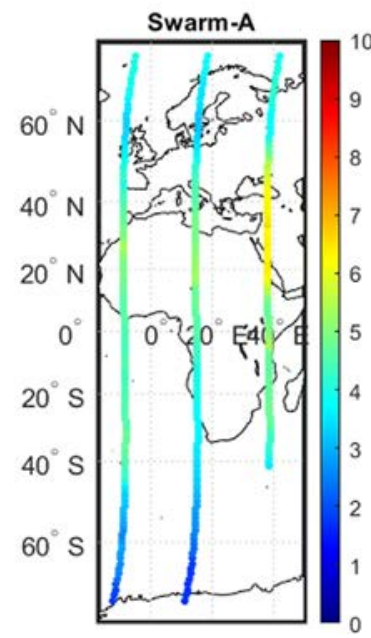

QD Asc | Year=2019, DOY =47 | LT=10:48
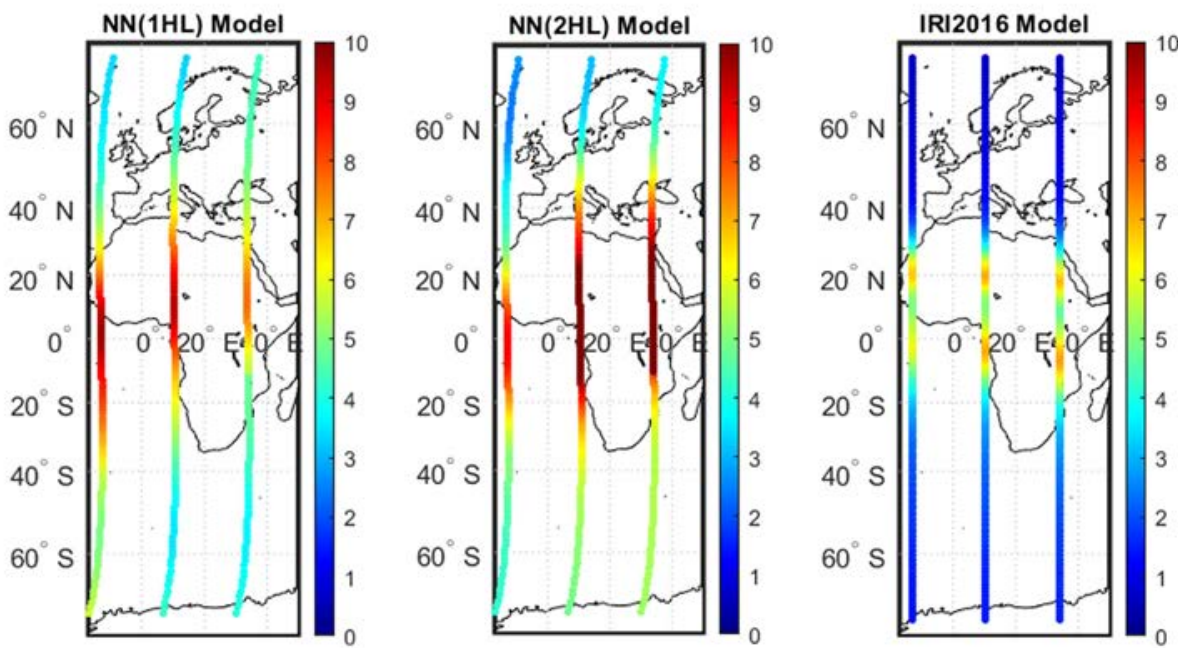

QD Asc $\mid$ Year=2019, DOY=137 | LT=02:43
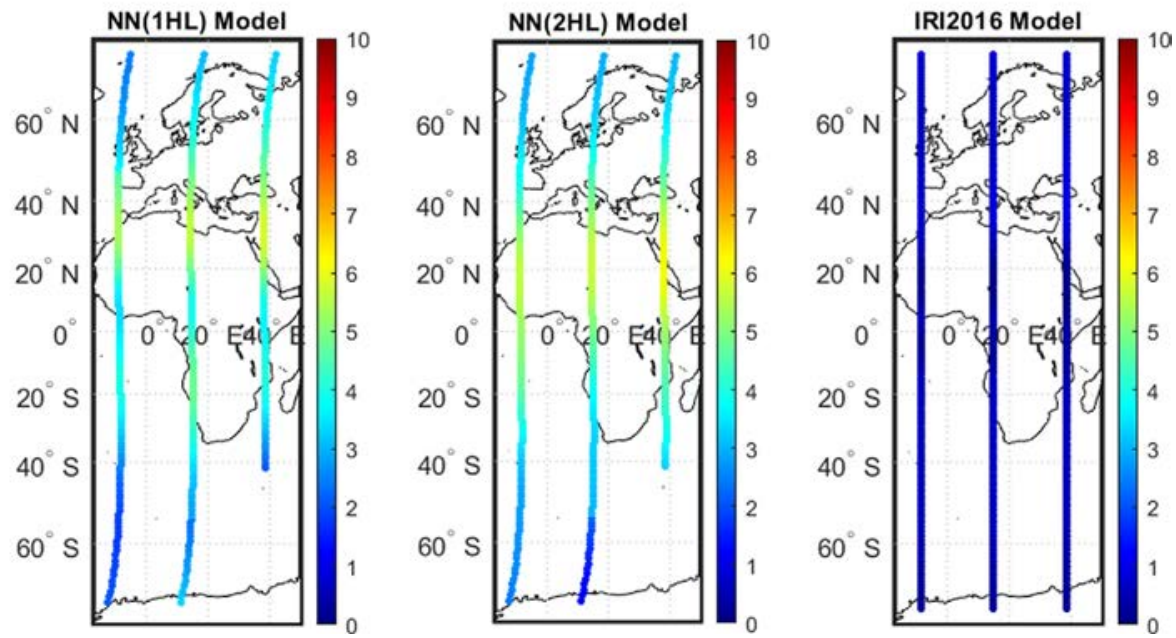

Fig. 6. Topside VTEC along the Swarm-A passes together with corresponding VTEC from the NN and IRI2016 models for Quiet Day Ascending passes (QD Asc). 
and the least variations ( 5 TECU) occurs between midnight and dawn.

For example, during the Sep equinox during 2019, the largest VTEC variations occur during the ascending trajectories with LT variations decreasing from 18:00 to 14:00 (afternoon). During the same period, the VTEC variations along the descending trajectories are 6 TECU with LT variations decreasing from 06:00 to 02:00 (pre-dawn). During the other periods of the year similar patterns, occur of large and small VTEC variations during the daytime and nighttime passes respectively.

Throughout the testing period, the VTEC variations predicted by the NN model are synchronized with the measured VTEC and follows the trend of the observations.

The performance of the four models is summarized in Fig. 4. This figure shows that the model that performed best was the one hidden layer model in the case of quiet days for descending trajectories model (QD Des (1HL)), with RMSE $=1.20$ TECU, and correlation coefficient $\mathrm{R}=0.76$. The worst performance as measured by RMSE occurred during the disturbed descending trajectories for one hidden layer model (DD Des (1HL)) with RMSE $=2.12$ TECU and $\mathrm{R}=0.54$. The worst performance as measured by correlation coefficient occurred during the disturbed descending trajectories for the two hidden layer model (DD Des (2HL) with $\mathrm{R}=0.47$ and $\mathrm{RMSE}=1.57$ TECU.

Fig. 5 shows scatter plots, linear regression lines and the correlation coefficients between the observed VTEC values derived from Swarm-A data and the predicted VTEC values from the $\mathrm{NN}(1 \mathrm{HL})$ and $\mathrm{NN}(2 \mathrm{HL})$ for the four models in the following order QD Asc, QD Des, DD Asc and DD Des.

In each case, the correlation coefficient as well as the equation for the linear regression line and the ideal prediction line $(\mathrm{y}=\mathrm{x})$ are given. The $\mathrm{NN}$ models underesti- (iii)

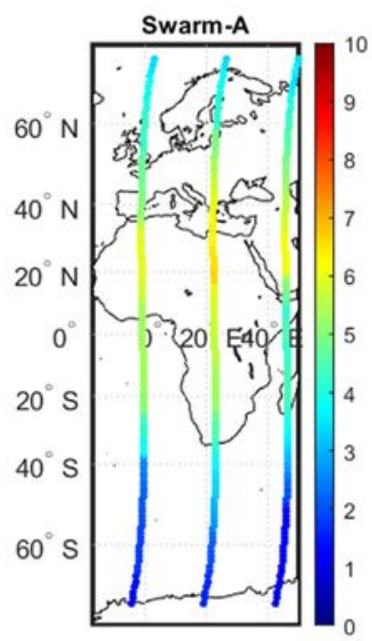

(iv)

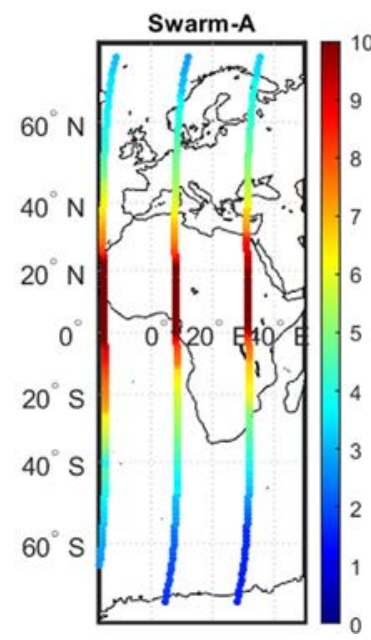

QD Asc | Year=2019, DOY=199 | LT=21:04
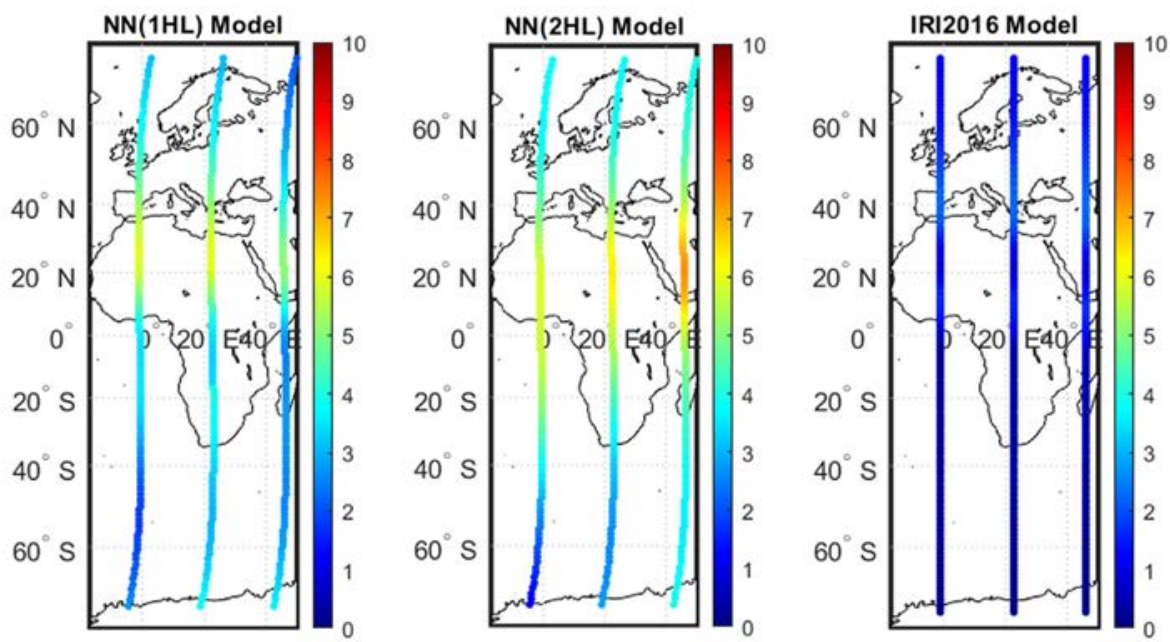

QD Asc | Year=2019, DOY=262 | LT=15:24
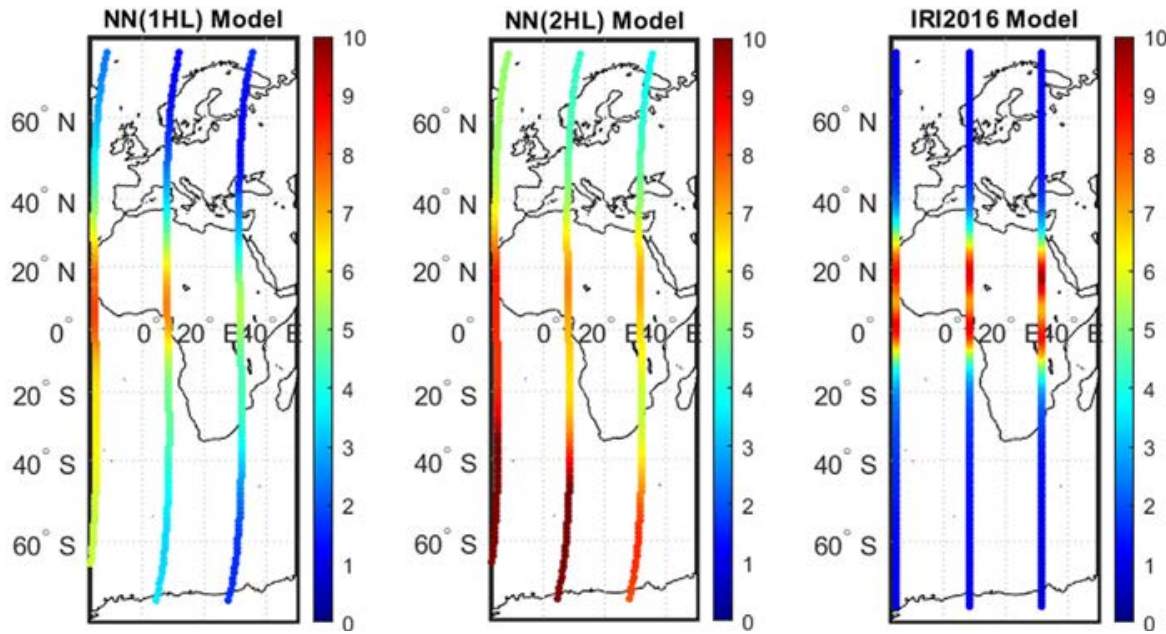

Fig 6. (continued) 
mate the measurements. The model with the best correlation coefficient is not the same as the model for which the linear regression is closest to the ideal prediction line. The best linear regression occurs with the DD Asc (1HL) model $(y=0.72 X+1.55)$ and the worst linear regression occurs with the DD Des (2HL) model $((y=0.38 X+2.95)$.

Figs. 6-9 show typical comparisons of topside VTEC from Swarm-A, NN and IRI2016 model for selected cases and passes of Swarm-A during the four periods of the year (March and September equinoxes, January and June solstices).

Each of the 16 panels of Figs. 6-9 show colour coded values of the measured VTEC along the tracks of
Swarm-A and the corresponding modelled VTEC derived from NN(1HL), NN(2HL) and IRI2016 models. The 16 panels are arranged according to four periods (the two equinoxes March and September, the two solstices January and June) each with examples of VTEC from the three models (NN(1HL), NN(2HL), and IRI2016) compared to Swarm-A VTEC. In each case, the comparisons are organized by DOY.

The VTEC derived from IRI2016 model was calculated along fixed longitudes namely the longitude where the Swarm-A paths crossed the geographic equator. The deviation of the Swarm path from this fixed longitude over the Euro-African sector is at most $2.6^{\circ}$ (due to the inclination of the Swarm-A orbit being $87.4^{\circ}$ ). (i)

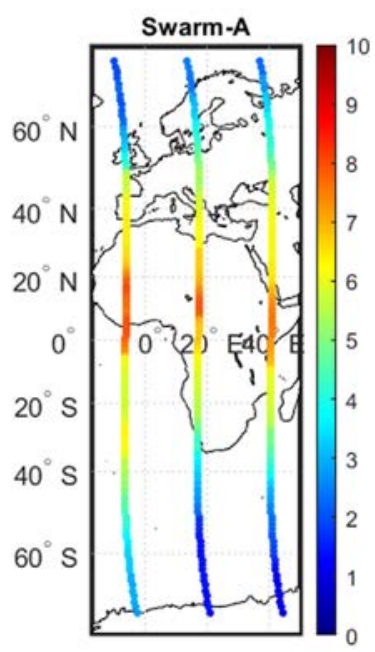

(ii)

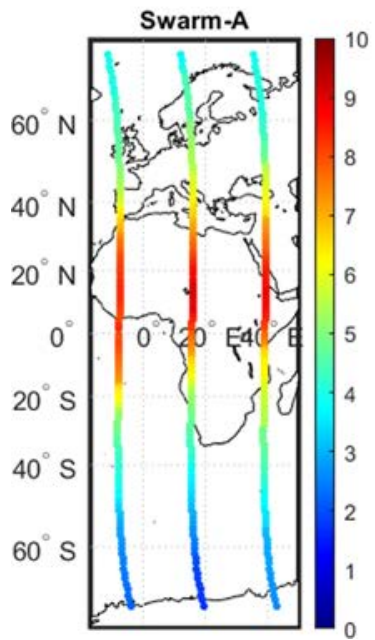

QD Des | Year=2019, DOY=50 | LT=22:29
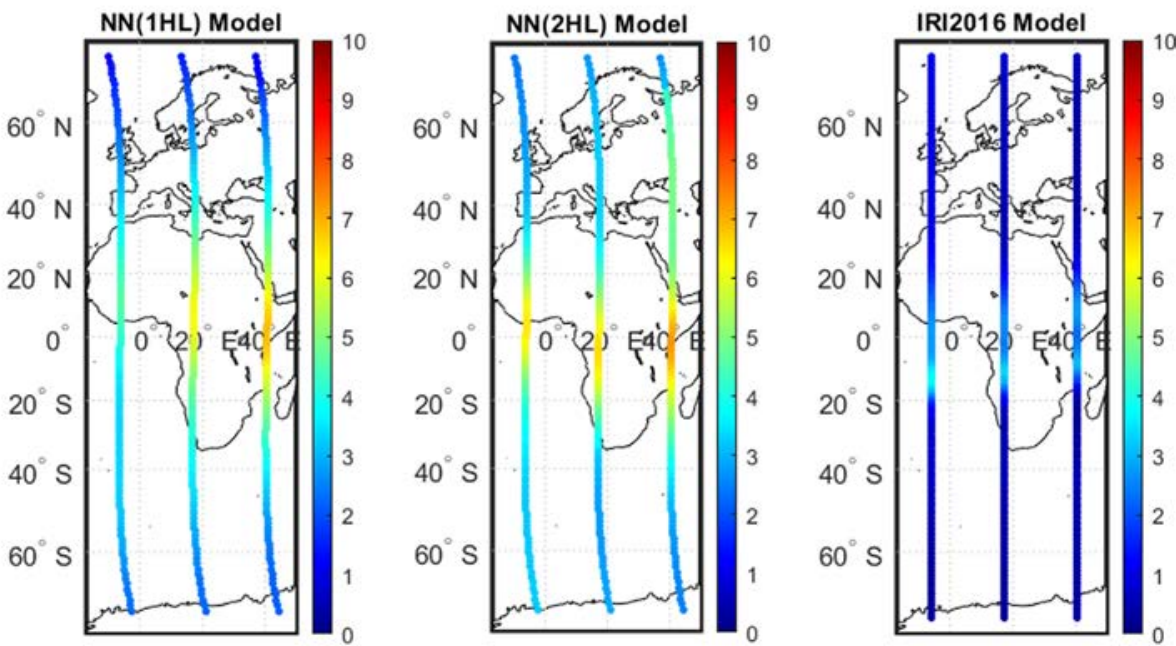

QD Des | Year=2019, DOY=142 | LT=14:13
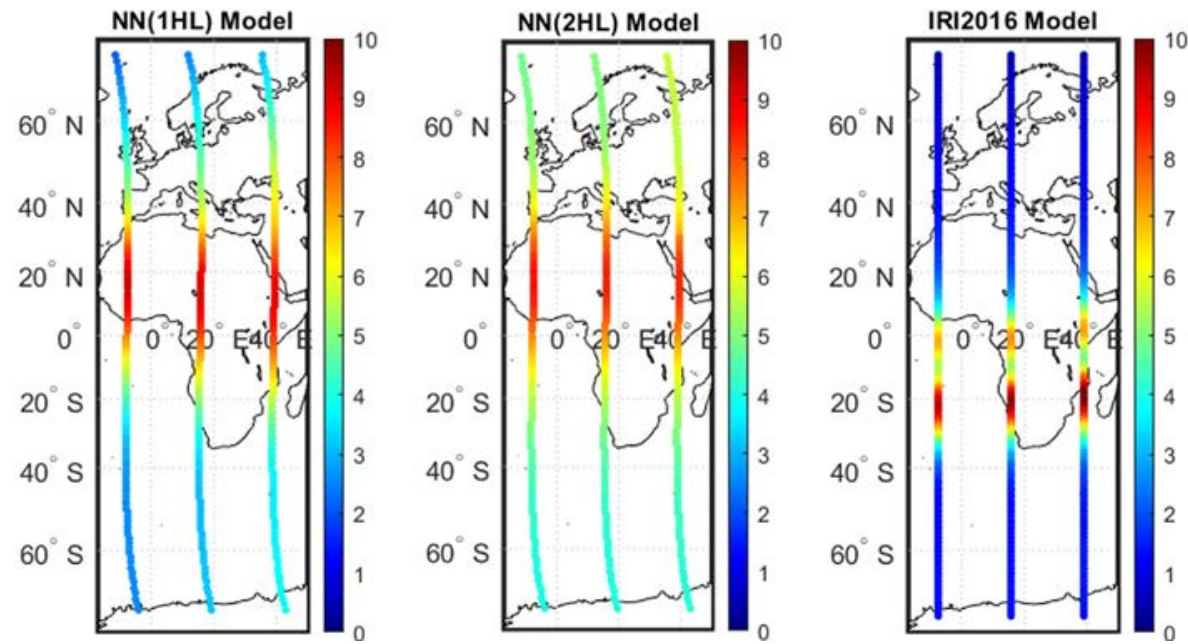

Fig. 7. Topside VTEC along the Swarm-A passes together with corresponding VTEC from the NN and IRI2016 models for Quiet Day Descending passes (QD Des). 


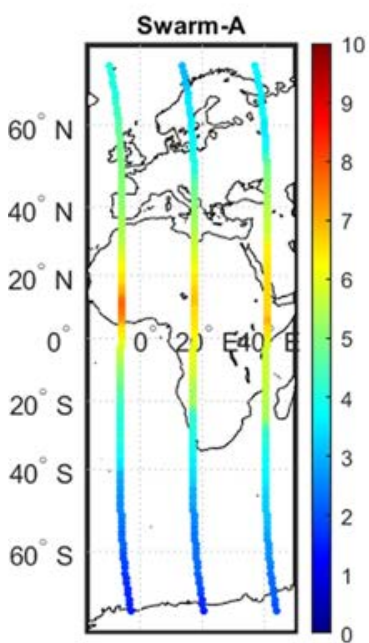

(iv)

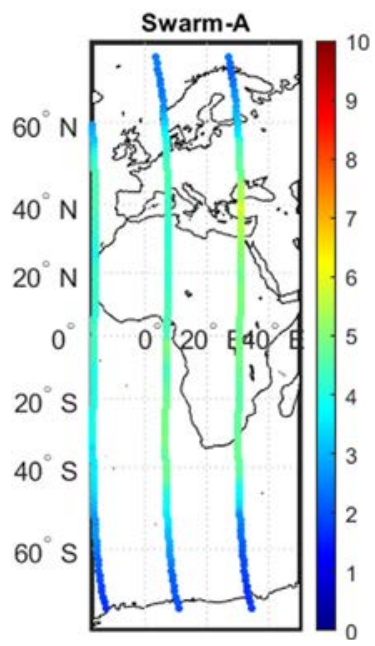

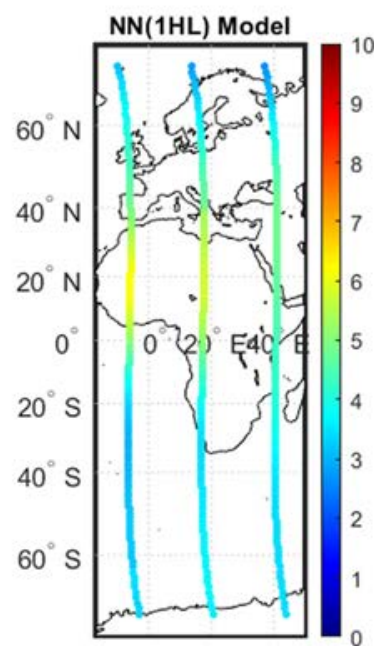
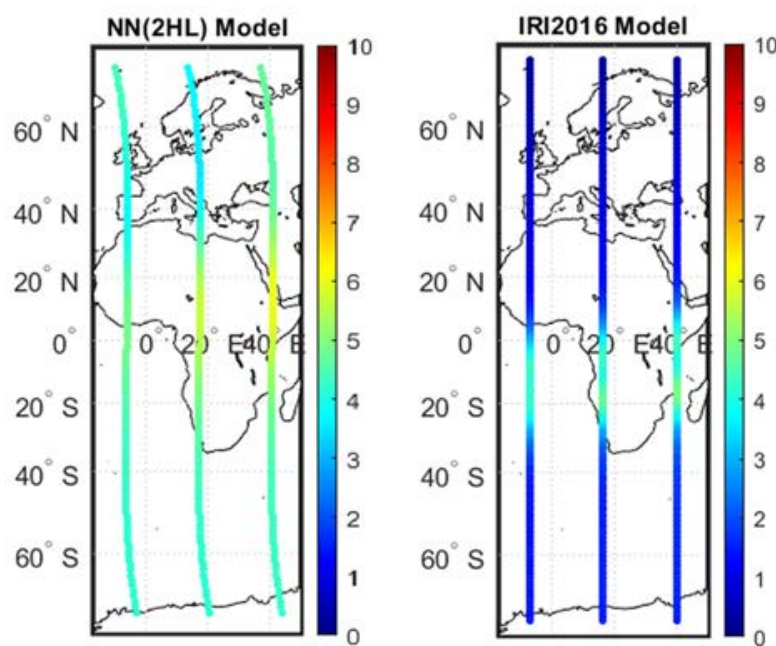

QD Des | Year=2019, DOY=257 | LT=03:54
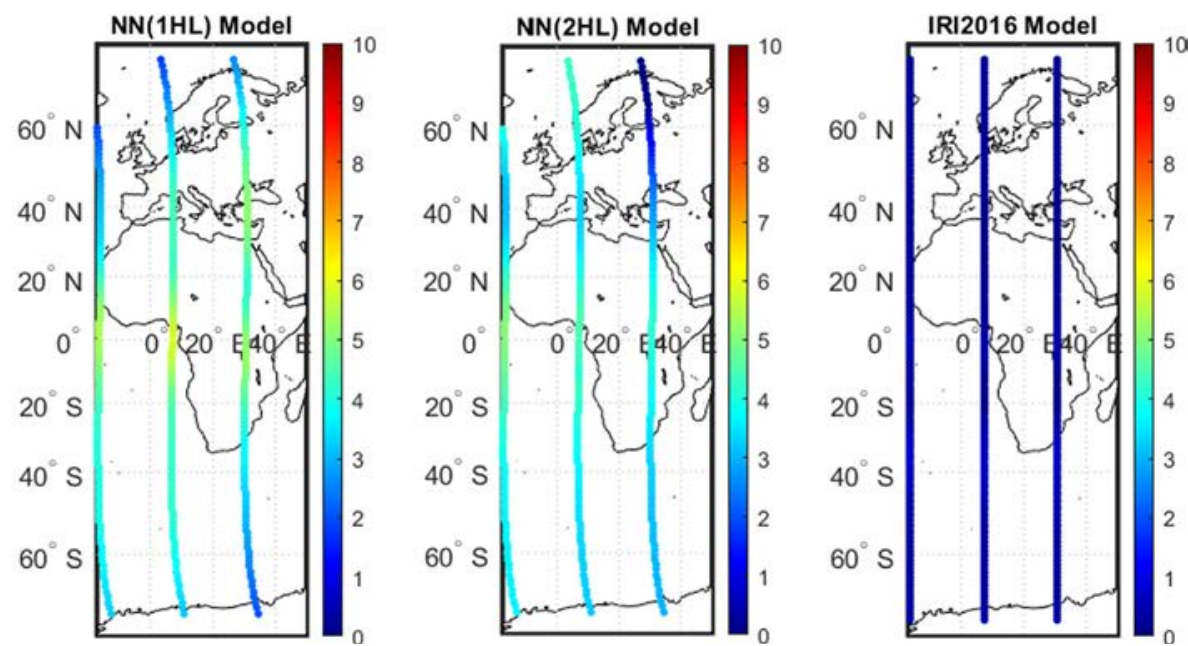

Fig 7. (continued)
The maps in Figs. 6-9 clearly display the dependence of the VTEC distribution on longitude and LT.

In all the selected cases, the NN models performed better than the IRI2016 model that frequently had an equatorial dip in the topside VTEC which was not matched by the Swarm-A measurements. The predictions of the IRI2016 model, in most cases, underestimates the Swarm-A VTEC.

The largest differences between the NN models and IRI2016 model occurred at midnight and during the early morning, while the differences were smaller during the daytime.

\subsection{The QD Asc model}

Fig. 6 illustrates the topside VTEC along the Swarm-A passes together with corresponding VTEC from the NN (QD Asc) and IRI2016 models.
Panel (i) of Fig. 6 shows the QD Asc daytime paths (LT = 10:48) during the Jan-Feb solstice (represented by DOY 47). Note that the best prediction of the VTEC for this day is along the central path over Africa $\left(10^{\circ}\right)$ where both models fit the Swarm-A measurements equally well. The NN (1HL) model matches the Swarm-A better than the NN (2HL) along the western longitude $\left(-13^{\circ}\right)$, while the reverse occurs at the eastern longitude $\left(34^{\circ}\right)$ where the NN (2HL) model predicts a better match with the measured VTEC than the NN (1HL). It is not clear why the two NN models have different performances in the eastern and western paths over Africa. The IRI2016 model is worse than the NN model because it shows two VTEC peaks on either side of the geomagnetic equator and underestimates the VTEC values at mid latitudes.

Panels (ii) and (iii) of Fig. 6 show the QD Asc nighttime paths ( $\mathrm{LT}=02: 43$ and $\mathrm{LT}=21: 04$ respectively) during the 
(i)

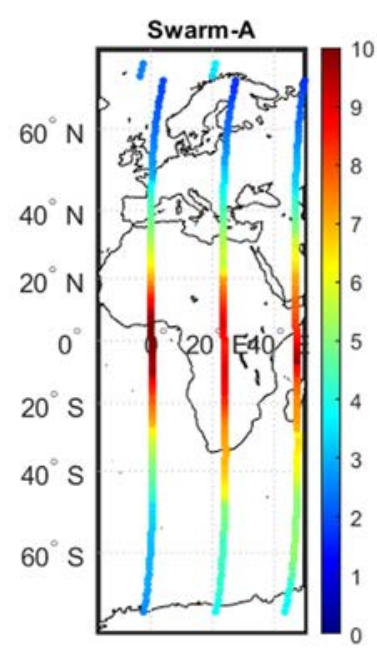

(ii)

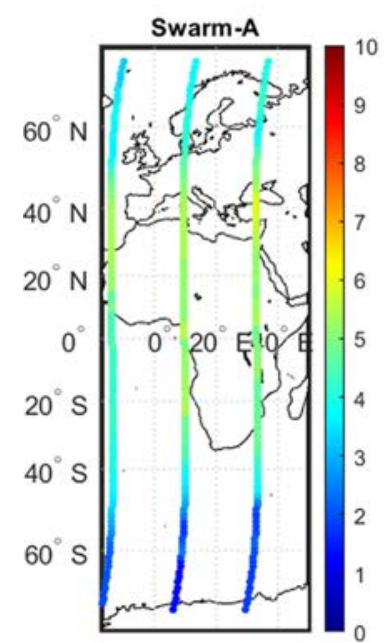

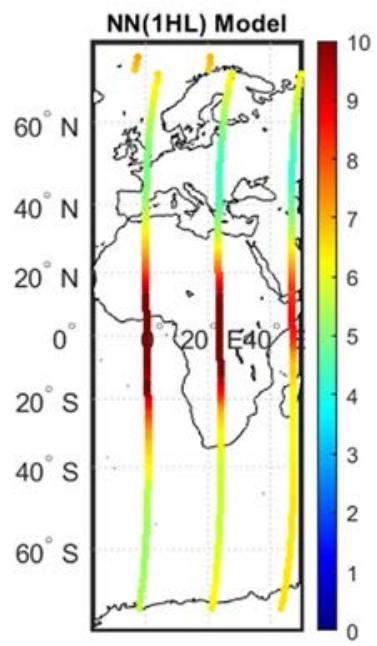
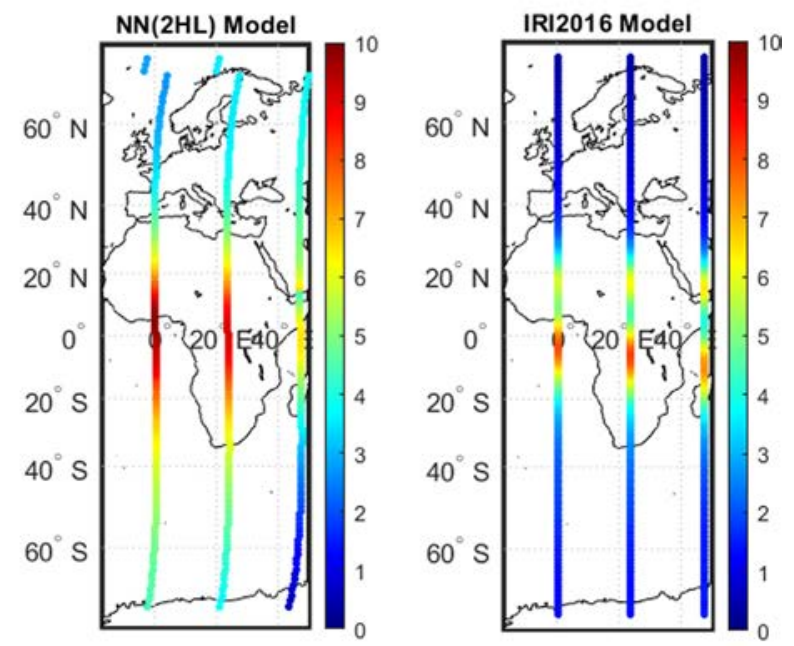

DD Asc | Year=2019, DOY=131 | LT=03:16
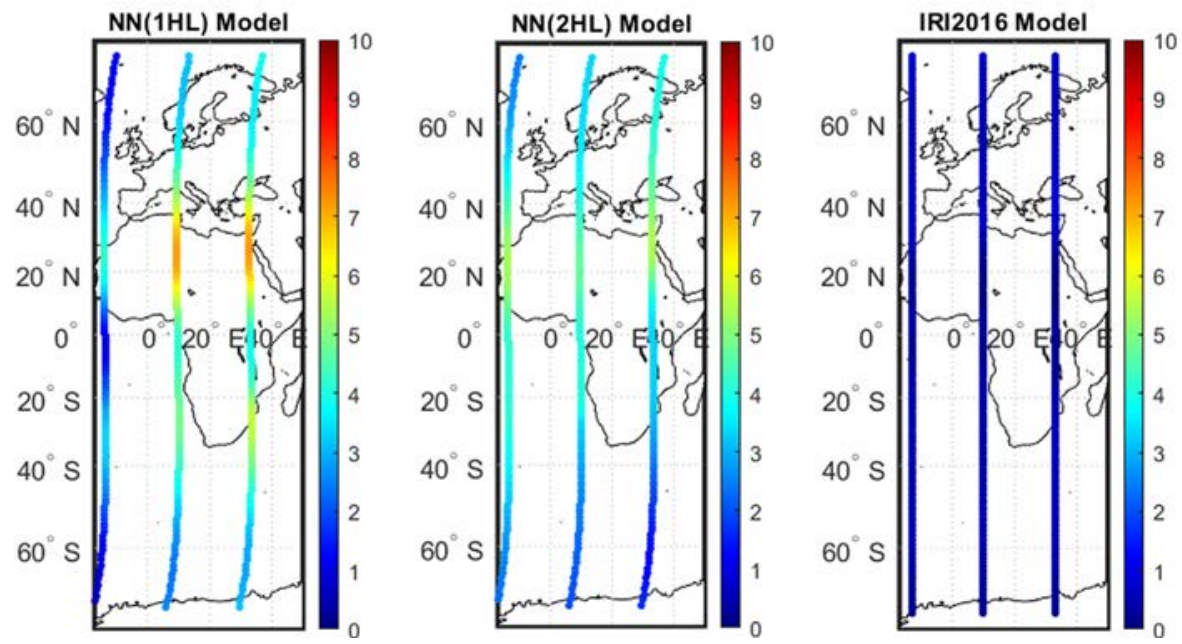

Fig. 8. Topside VTEC along the Swarm-A passes together with corresponding VTEC from the NN and IRI2016 models for Disturbed Day Ascending passes (DD Asc).

Mar-May equinox (represented by DOY 137) and the JunAug solstice (represented by DOY 199). The predictions of the VTEC for these days by the NN (1HL) and NN (2HL) models closely match the Swarm-A measurements over Africa. Note the hemispheric asymmetry of VTEC; VTEC above 6 TECU only occurred on northern hemisphere mid latitudes on these days. The IRI2016 model predicts VTEC $<2$ TECU during all 3 paths during these days. Thus significantly underestimating Swarm VTEC.

Panel (iv) of Fig. 6 shows the QD Asc daytime paths $(\mathrm{LT}=15: 24)$ during the Sep equinox (represented by DOY 262). Note that the best prediction of the VTEC for this day is by the NN (1HL) model along the western path over Africa $\left(-15^{\circ}\right)$.
The IRI2016 model predicted almost identical doublepeak VTEC distributions for all 3 paths over Africa and underestimated the measured values by 3 TECU.

\subsection{The QD Des model}

Fig. 7 illustrates the topside VTEC along the Swarm-A passes together with corresponding VTEC from the NN (QD Des) and IRI2016 models.

Panel (i) of Fig. 7 shows the QD Des nighttime paths $(\mathrm{LT}=22: 29)$ during the Jan-Feb solstice (represented by DOY 50). Note that the both The NN (1HL) and the NN (2HL) models give good predictions of the VTEC for this day along the eastern path over Africa $\left(41^{\circ} \mathrm{E}\right)$ as the VTEC values closely match that of Swarm. On the 
(iii)

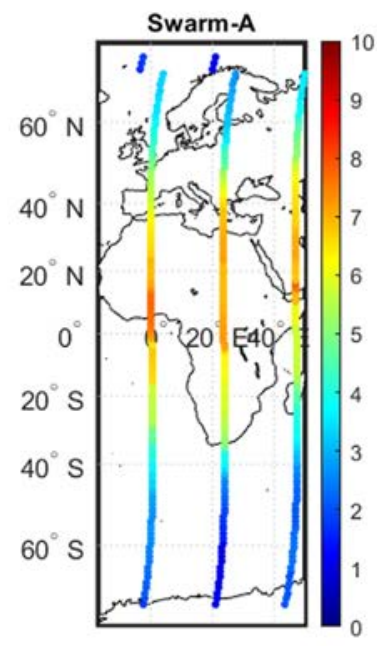

(iv)

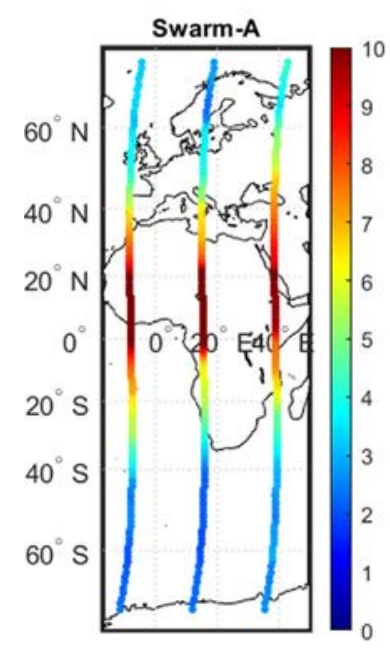

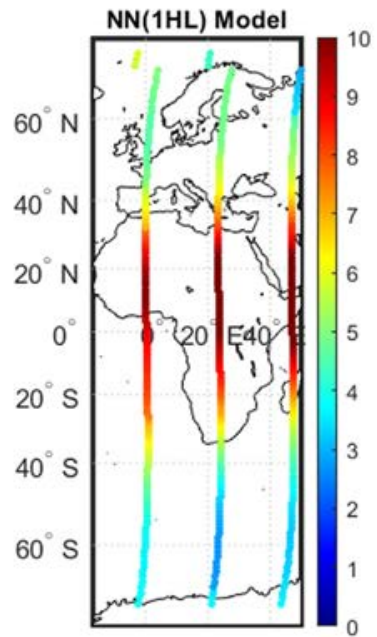
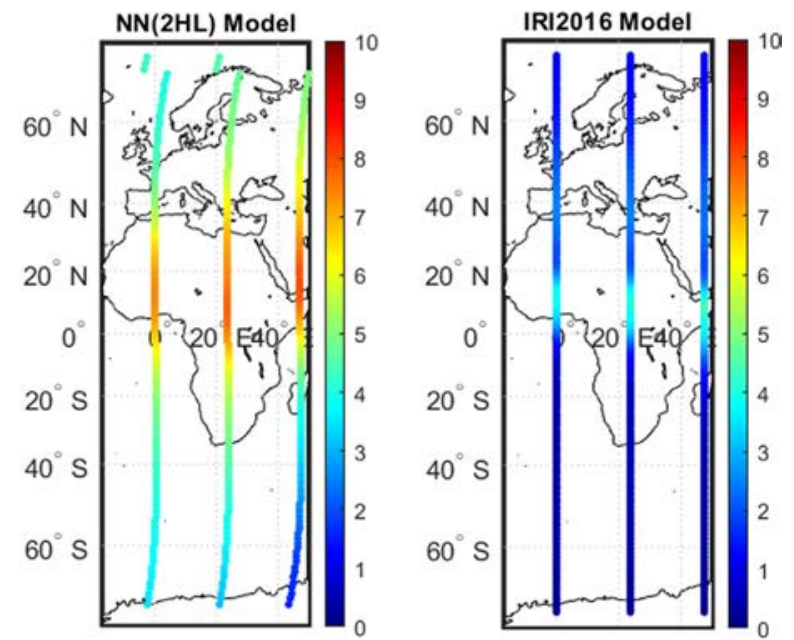

DD Asc | Year=2019, DOY=244 | LT=17:01
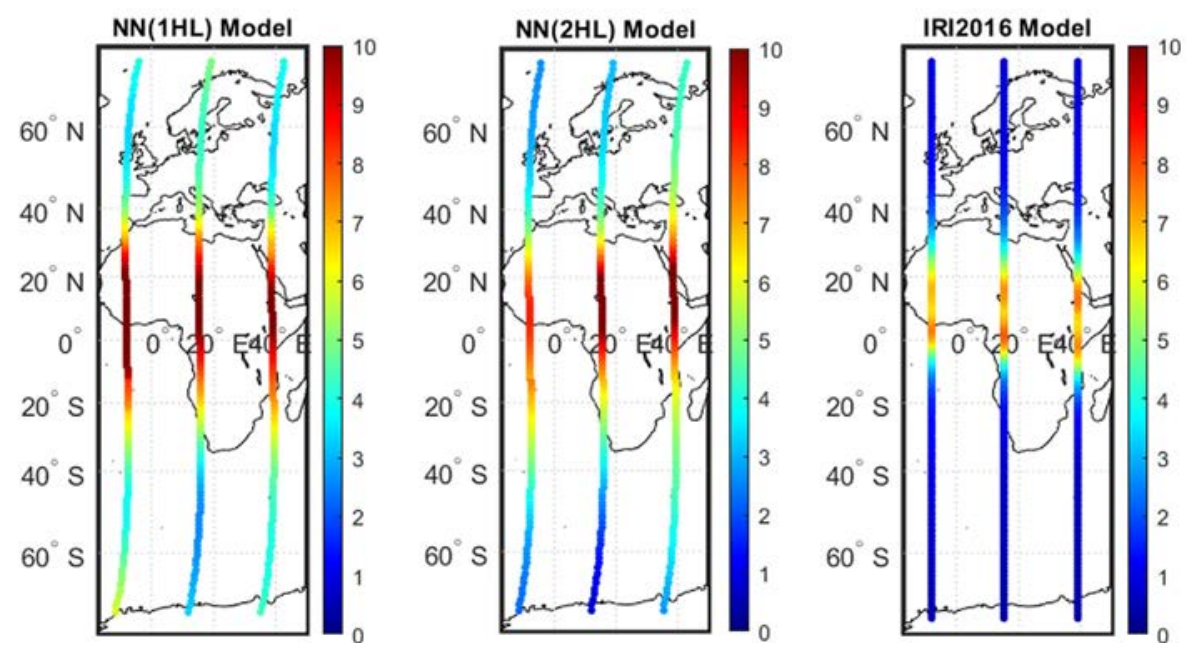

Fig 8. (continued)

other two paths of Swarm-A the NN (2HL) model gives slightly better estimates of VTEC values than the NN (1HL) model. The IRI2016 model predicts VTEC values lower than half of the measured values during all 3 paths during this day. During all 3 Swarm-A paths the peaks of VTEC seem to occur at the geomagnetic equator $\left(10^{\circ}\right.$ $\mathrm{N}$ geographic), whereas the $1 \mathrm{HL}$ and $2 \mathrm{HL}$ models predict peak at around the geographic equator.

Panels (ii) of Fig. 7 shows the QD Des daytime paths $(\mathrm{LT}=14: 13)$ during the Mar-May equinox (represented by DOY 142). The predictions of the VTEC for this day by the NN (1HL) and NN (2HL) models closely match the Swarm-A measurements over Africa. At this LT the longitudinal variations of the VTEC is minimal. The IRI2016 predicts 2 VTEC peaks on all 3 paths over Africa, one peak at the geomagnetic equator and the other peak in the southern hemisphere at about $20^{\circ} \mathrm{S}$ (geographic), while Swarm VTEC shows an extended peak covering latitudes from about -10 to $30^{\circ}$.

Panels (iii) of Fig. 7 shows the QD Des daytime paths $(\mathrm{LT}=10: 50)$ during the Jun-Aug solstice (represented by DOY 180). The predictions of the VTEC for this day by the NN (1HL) and NN (2HL) models are similar to the Swarm-A measurements over Africa. The IRI2016 model predicts the peaks of the VTEC to be in the southern hemisphere during all 3 paths during this day that is inconsistent with the Swarm-A measurements.

Panel (iv) of Fig. 7 shows the QD Des nighttime paths $(\mathrm{LT}=03: 54)$ during the Sep equinox (represented by DOY 257). Note that the predictions of the VTEC for this day by the NN (1HL) and NN (2HL) models are similar to the Swarm-A measurements over Africa and they have low values ( $\leq 5$ TECU). The IRI2016 model underestimates the measured values by 5 TECU. 


\subsection{The DD Asc model}

Fig. 8 illustrates the topside VTEC along the Swarm-A passes together with corresponding VTEC from the NN (DD Asc) and IRI2016 models.

Panel (i) of Fig. 8 shows the DD Asc daytime paths $(\mathrm{LT}=14: 34)$ during the Jan-Feb solstice (represented by DOY 5). Note that the NN (2HL) model provides the best prediction of the VTEC for this day. The NN (1HL) model overestimates the VTEC values along the central and eastern longitudes $\left(24^{\circ}\right.$ and $0^{\circ}$ respectively). The IRI2016 model predicts VTEC values lower than the measured values during all 3 paths during this day. In addition, the IRI2016 shows 2 VTEC peaks, the largest of which occurs at $10^{\circ} \mathrm{S}$ geographic, whereas the Swarm only show one VTEC peak.
Panels (ii) of Fig. 8 shows the DD Asc nighttime paths $(\mathrm{LT}=03: 16)$ during the Mar-May equinox (represented by DOY 131). Note that the best prediction of the VTEC for this day is by the NN (2HL) model. The NN (1HL) model overestimates the VTEC Swarm-A values along the central and eastern longitudes $\left(33^{\circ}\right.$ and $10^{\circ}$ respectively). The IRI2016 predicts VTEC below 1 TECU in all paths, thus significantly underestimating Swarm VTEC.

Panels (iii) of Fig. 8 shows the DD Asc daytime paths $(\mathrm{LT}=19: 22)$ during the Jun-Aug solstice (represented by DOY 218). Note that the NN (2HL) model provides the best prediction of the VTEC for this day. The NN (1HL) model over estimates the VTEC Swarm-A values along all 3 longitudes. The IRI2016 model predicts VTEC values lower than half of the measured values during all 3 paths during this day. (i)

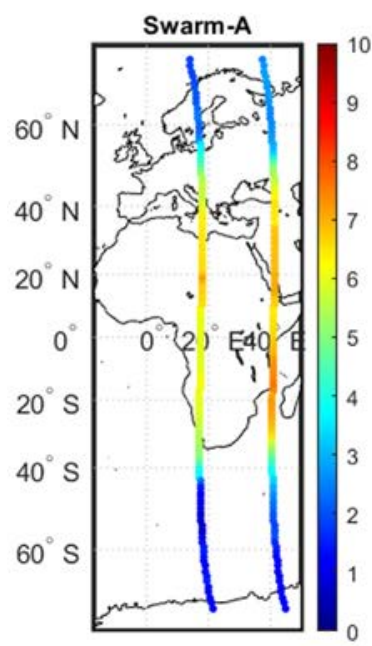

(ii)

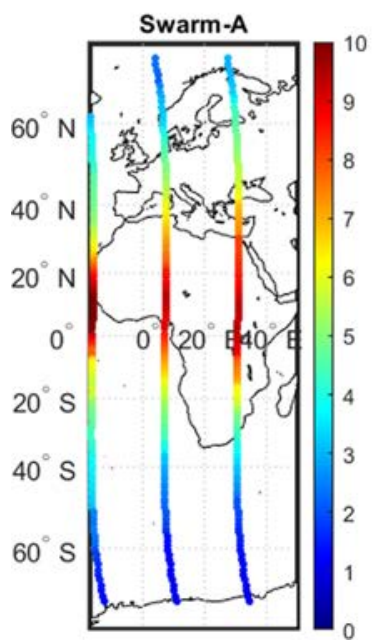

DD Des | Year=2019, DOY=31 | LT=00:20
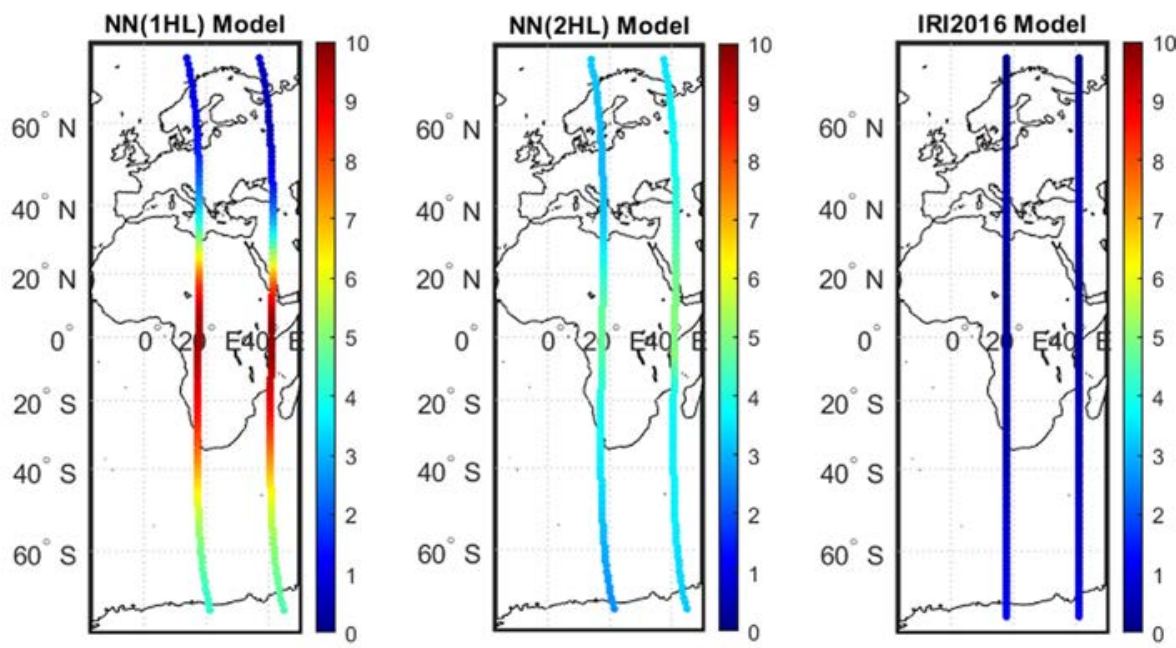

DD Des | Year=2019, DOY=95 | LT=18:27
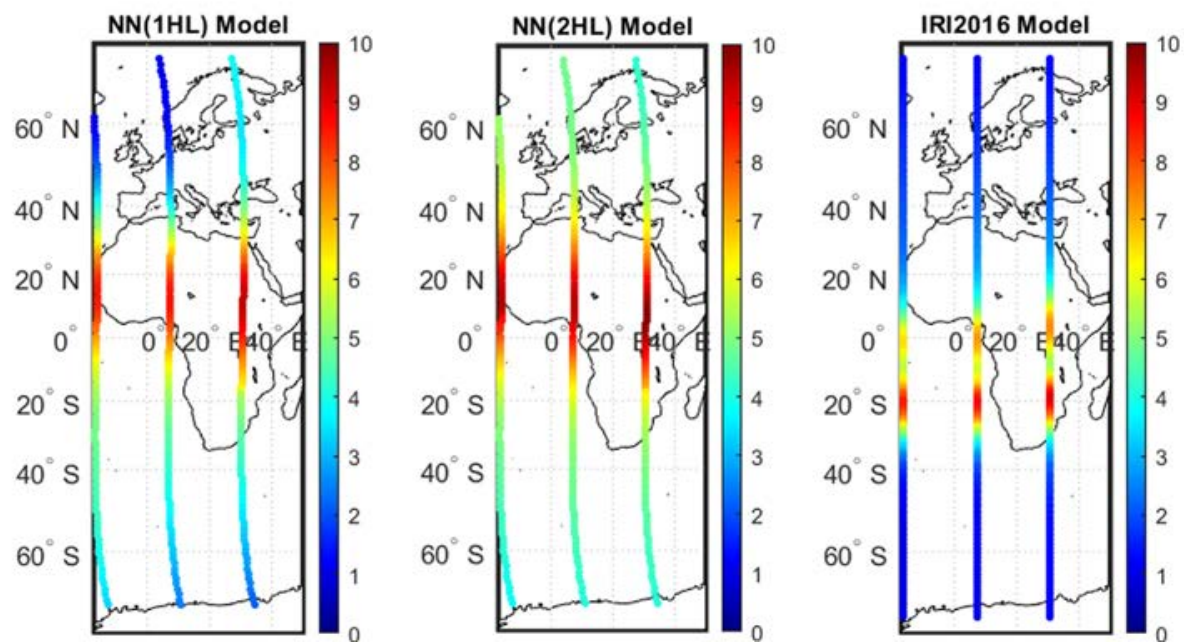

Fig. 9. Topside VTEC along the Swarm-A passes together with corresponding VTEC from the NN and IRI2016 models for Disturbed Day Descending passes (DD Des). 
(iii)

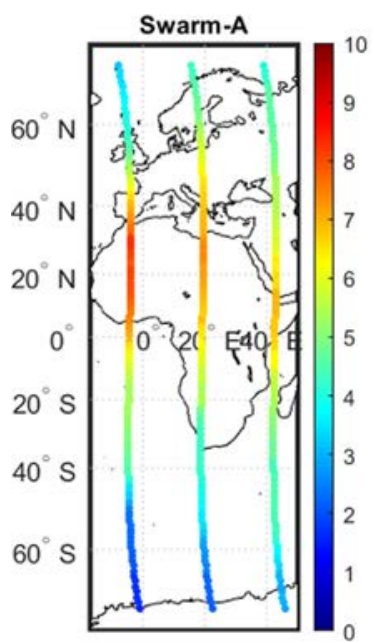

(iv)

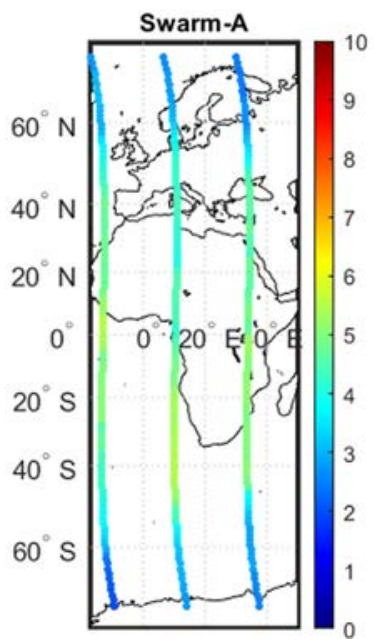

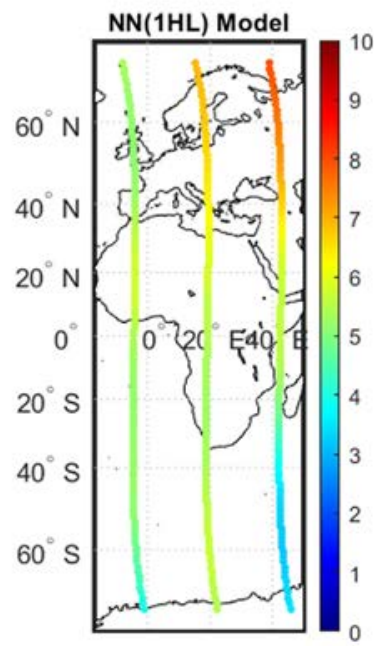
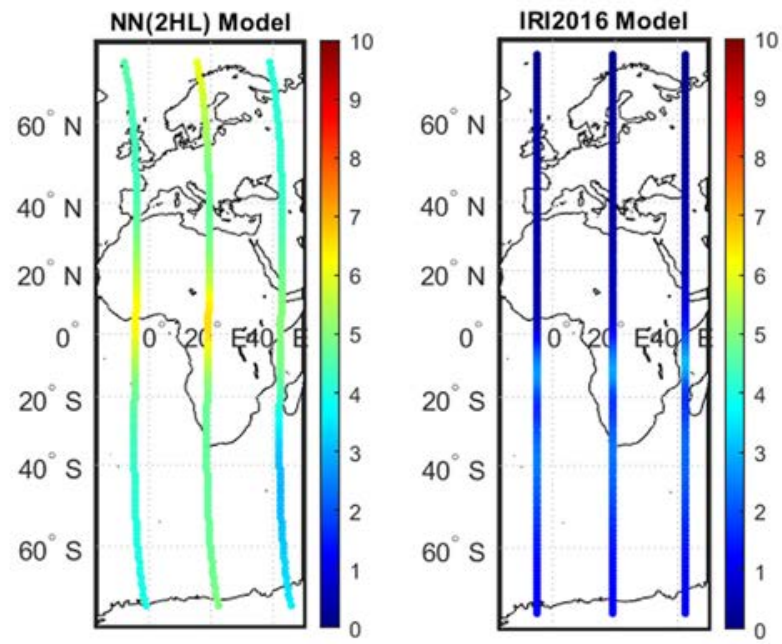

DD Des $\mid$ Year =2019, DOY $252 \mid \mathrm{LT}=04: 22$
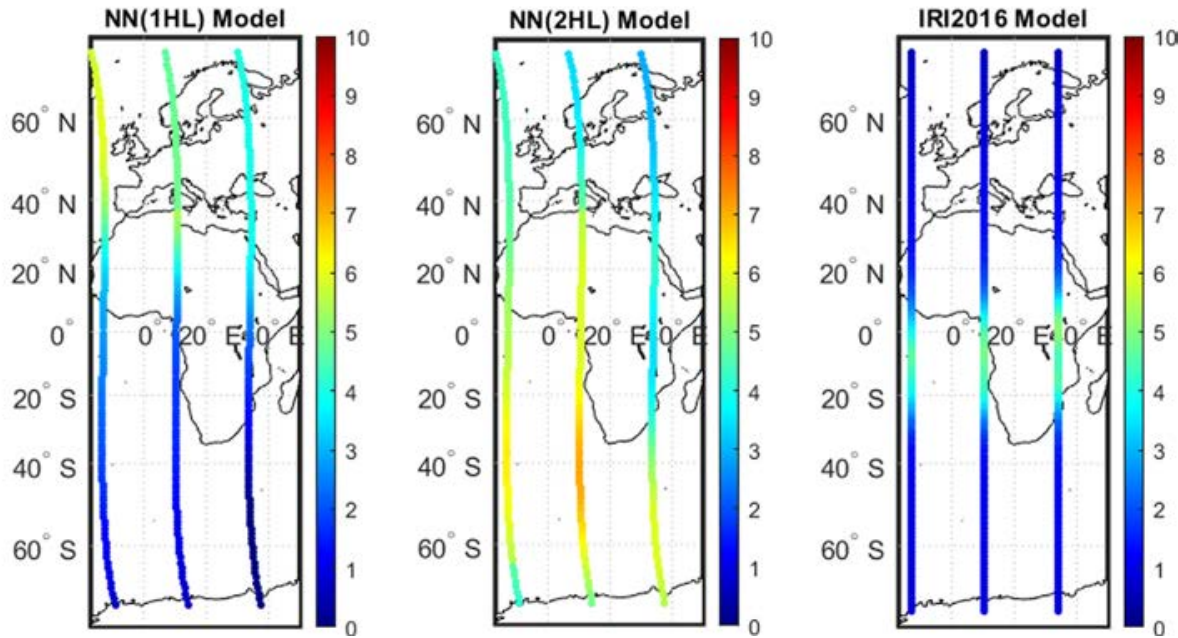

Fig 9. (continued)

Panel (iv) of Fig. 8 shows the DD Asc nighttime paths $(\mathrm{LT}=17: 01)$ during the Sep equinox (represented by DOY 244). The predictions of the VTEC for this day by the NN (1HL) and NN (2HL) models closely match the Swarm-A measurements over Africa. The IRI2016 model underestimates the measured values by 4 TECU and erroneously predicts two VTEC peaks in the northern hemisphere during all 3 paths during this day.

\subsection{The DD Des model}

Fig. 9 illustrates the topside VTEC along the Swarm-A passes together with corresponding VTEC from the NN (DD Des) and IRI2016 models.
Panel (i) of Fig. 9 shows the DD Des nighttime paths ( $\mathrm{LT}=00: 20)$ during the Jan-Feb solstice (represented by DOY 31). Note that the NN (2HL) model gives the best prediction with RMSE $=1.99$ TECU, while the NN (1HL) model overestimates the VTEC values with $\mathrm{RMSE}=2.87$ TECU. The IRI2016 model greatly underestimates Swarm VTEC as it over both paths.

Panel (ii) of Fig. 9 shows the DD Des at the dusk paths ( $\mathrm{LT}=18: 46$ ) during the Mar-May equinox (represented by DOY 95). The predictions of the VTEC for this day by the NN (1HL) and NN (2HL) models closely match the Swarm-A measurements over Africa. At this LT the longitudinal variations of the VTEC is minimal. The IRI2016 predicts 2 VTEC peaks on all 3 paths over Africa, one peak at the geographic equator and the other peak in the south- 
Table 2

The RMSE for all results in Figs. 6-9. All RMSE values are expressed in TECU

\begin{tabular}{|c|c|c|c|c|c|c|c|c|c|c|c|c|}
\hline & \multicolumn{3}{|c|}{ Jan-Feb Solstice } & \multicolumn{3}{|c|}{ Mar-May Equinox } & \multicolumn{3}{|c|}{ Jun-Aug Solstice } & \multicolumn{3}{|c|}{ Sep Equinox } \\
\hline & NN (1HL) & $\mathrm{NN}(2 \mathrm{HL})$ & IRI2016 & NN 1HL & $\mathrm{NN} 2 \mathrm{HL}$ & IRI2016 & NN 1HL & $\mathrm{NN} 2 \mathrm{HL}$ & IRI & NN $1 \mathrm{HL}$ & $\mathrm{NN} 2 \mathrm{HL}$ & IRI \\
\hline QD Asc & 1.45 & 1.84 & 2.96 & 0.86 & 0.56 & 3.87 & 1.22 & 0.89 & 3.58 & 2.65 & 4.12 & 2.90 \\
\hline QD Des & 1.66 & 1.46 & 4.17 & 0.79 & 0.80 & 2.79 & 1.01 & 1.19 & 2.79 & 0.76 & 1.26 & 3.71 \\
\hline DD Asc & 1.78 & 1.55 & 3.13 & 1.34 & 0.90 & 3.96 & 2.20 & 0.97 & 3.67 & 1.49 & 1.41 & 3.62 \\
\hline DD Des & 2.87 & 1.99 & 4.89 & 1.34 & 1.43 & 2.36 & 1.55 & 1.41 & 4.22 & 2.43 & 1.45 & 2.56 \\
\hline
\end{tabular}

ern hemisphere at about $20^{\circ} \mathrm{S}$, while Swarm only observed one VTEC peak.

Panels (iii) of Fig. 9 shows the DD Des daytime paths ( $\mathrm{LT}=09: 84$ ) during the Jun-Aug solstice (represented by DOY 191). The predictions of the VTEC for this day by the NN (1HL) and NN (2HL) models are very different from the Swarm-A measurements over Africa.

Panel (iv) of Fig. 9 shows the DD Des nighttime paths $(\mathrm{LT}=04: 37$ ) during the Sep equinox (represented by DOY 252). Note that the predictions of the VTEC for this day by the NN (2HL) model is much better than the NN (1HL) model. The NN(2HL) model is generally better than the NN(1HL) model for disturbed days (except for the Mar-May equinox) probably because the extra layer in the $\mathrm{NN}(2 \mathrm{HL})$ model improves the ability of the model to handle the complexity of the ionosphere during the disturbed days. The peaks of the IRI2016 model matches the measured VTEC at the geographic equator.

The best performance of the NN models occurred at different local times, but in all cases during the equinox months: The QD Asc NN (2HL) model performed best during the March equinox at $\mathrm{LT}=02.43(\mathrm{RMSE}=0.56$ TECU), the QD Des NN (1HL) model performed best during the September equinox at $\mathrm{LT}=15.41(\mathrm{RMSE}=0.76$ TECU), the DD Asc NN (2HL) model gave the best performance during the March equinox at $\mathrm{LT}=3.26$ $(\mathrm{RMSE}=0.90 \mathrm{TECU})$, and the DD Des NN (1HL) model also performed best during the March equinox, but at $\mathrm{LT}=10.95(\mathrm{RMSE}=1.34 \mathrm{TECU})$. All local times were covered by the Swarm-A satellite over the four years of training data.

Table 2 shows a summary of the RMSE for all results in Figs. 6-9 organized by period of observation and NN models.

\section{Conclusion}

The NN models presented here is the first such attempt at comparing NN models for the topside VTEC based on Swarm-A measurements.

The NN trained on Swarm-A measurements of VTEC and geophysical inputs provided useful predictions of the topside VTEC. The single hidden layer NN performed better in the case of quiet days for both ascending and descending trajectories, while the double hidden layer $\mathrm{NN}$ performed better in the case of disturbed days for both trajectories. This is true when looking at both RMSE and correlation. These results might imply that the relation between the input parameters and the VTEC values during the disturbed days is more complex and hence the NN may need an additional hidden layer to be able to converge to a smaller error than that which was obtained with the $1 \mathrm{HL}$ and 2HL models.

The 11 input parameters (DOYs, DOYc, HRs, HRc, latitude, longitude, Dst, F10.7, PF $10 \mathrm{MeV}$, PF $30 \mathrm{MeV}$, and $\mathrm{PF} 60 \mathrm{MeV}$ ) proved to be adequate. We investigated the Ap index as an additional input parameter, but it gave worse results.

Comparisons of the NN models with the topside VTEC calculated by means of the IRI2016 model, with measured F10.7 as optional input, demonstrated that both the NN models in all cases performed significantly better than the IRI2016 model. The IRI model frequently had an equatorial dip in the topside VTEC that was not matched by the Swarm-A measurements.

Future work will include testing the contribution of each of the input parameters to the accuracy of the predictions. The use of additional hidden layers to improve the NN models for disturbed days (DD Asc and DD Des) will be investigated. Validations will be performed with other instruments including incoherent scattering radars.

The validation period corresponded to a low solar activity. In future, a more comprehensive validation may be performed by selecting periods that represent both high and low solar activity.

\section{Declaration of Competing Interest}

The authors declare that they have no known competing financial interests or personal relationships that could have appeared to influence the work reported in this paper.

\section{Acknowledgment}

The authors acknowledge the ESA Swarm team for the Swarm mission and for providing the Swarm data via the server for Swarm data distribution (ftp://Swarm-diss.eo.esa.int). The WDC team in Kyoto are acknowledged for maintaining the Most Quiet and Disturbed days of each month via the website (http://wdc.kugi.kyoto-u.ac.jp/qddays/index.html). The OMNI web is acknowledged for providing the Ap and Dst indices and the F10.7 solar radio flux, and solar proton data on the website (http://omniweb.gsfc.nasa.gov/). We acknowledge the Community Coordinated Modeling Center 
(CCMC) through their public Runs on Request system (http://ccmc.gsfc.nasa.gov for the IRI2016 Model. The IRI2016 online was developed by the (Dieter Bilitza and IRI team).

\section{References}

Ajabshirizadeh, A., Jouzdani, N.M., Abbassi, S., 2011. Neural network prediction of solar cycle 24. Res. Astron. Astrophys. 11 (4), 491.

Baboo, S.S., Shereef, K.I., 2010. An efficient weather forecasting system using artificial neural network. Int. J. Environ. Sci. Technol. 1 (4), 321326.

Bakr, M.H., Negm, M.H., 2012. Modeling and design of high-frequency structures using artificial neural networks and space mapping. Advances in Imaging and Electron Physics, vol. 174. Elsevier, pp. 223-260.

Belehaki, A., Tsagouri, I., 2002. Investigation of the relative bottomside/topside contribution to the total electron content estimates. Ann. Geophys. 45 (1), 73-86. https://doi.org/10.4401/ag-3498.

Bortnik, J., Chu, X., Ma, Q., Li, W., Zhang, X., Thorne, R.M., Spence, H. E., 2018. Artificial neural networks for determining magnetospheric conditions. In: Machine Learning Techniques for Space Weather. Elsevier, pp. 279-300.

Bothmer, V., Daglis, I.A., 2007. Space Weather: Physics and Effects, 213, 315-318, 438 pp., Praxis Publishing Ltd., Chichester, UK.

Bilitza, D., 2009. Evaluation of the IRI-2007 model options for the topside electron density. Adv. Space Res. 44 (6), 701-706. https://doi.org/ 10.1016/j.asr.2009.04.036.

Buonsanto, M.J., 1999. Ionospheric storms - a review. Space Sci. Rev. 88 (3-4), 563-601.

Cander. R., 1998. Artifficial NN Applications in ionospheric studies.

Coïsson, P., Radicella, S.M., Nava, B., 2002. Comparisons of experimental topside electron concentration profiles with IRI and NeQuick models. Ann. Geophys. 45 (1).

Depuev, V.H., Pulinets, S.A., 2004. A global empirical model of the ionospheric topside electron density. Adv. Space Res. 34 (9), 2016 2020.

Ercha, A., Zhang, D., Ridley, A.J., Xiao, Z., Hao, Y., 2012. A global model: Empirical orthogonal function analysis of total electron content 1999-2009 data. J. Geophys. Res. 117, A03328. https://doi. org/10.1029/2011JA017238.

Fiori, R.A.D., Boteler, D.H., Burchill, J., Koustov, A.V., Cousins, E.D.P., Blais, C., 2013. Potential impact of Swarm electric field data on global 2D convection mapping in combination with SuperDARN radar data. J. Atmos. Sol.-Terr. Phys. 93, 87-99. https://doi.org/10.1016/ j.jastp.2012.11.013.

Fiori, R.A.D., Boteler, D.H., Koustov, A.V., Knudsen, D., Burchill, J.K., 2014. Investigation of localized $2 \mathrm{D}$ convection mapping based on artificially generated Swarm ion drift data. J. Atmos. Sol. Terr. Phys. 114, 30-41. https://doi.org/10.1016/j.jastp.2014.04.004.

Fuller-Rowell, T., Codrescu, M., Moffett, R., Quegan, S., 1994. Response of the thermosphere and ionosphere to geomagnetic storms. J. Geophys. Res.: Space Phys. 99 (A3), 3893-3914.

Fuller-Rowell, T., Codrescu, M., Wilkinson, P., 2000. Quantitative modelling of the ionospheric response to geomagnetic activity. Ann. Geophys. 18 (7), 766-781.

Habarulema, J.B., McKinnell, L.-A., Cilliers, P.J., Opperman, B.D.L., 2009. Application of neural networks to South African GPS TEC modelling. Adv. Space Res. 43 (11), 1711-1720. https://doi.org/ 10.1016/j.asr.2008.08.020.

Habarulema, J.B., 2010. A contribution to TEC modelling over Southern Africa using GPS data PhD Thesis. Rhodes University.

Habarulema, J.B., McKinnell, L.-A., Opperman, B.D., 2011. Regional GPS TEC modeling: Attempted spatial and temporal extrapolation of TEC using neural networks. J. Geophys. Res. 116, A04314. https://doi. org/10.1029/2010JA016269.
Habarulema, J.B., McKinnell, L.-A., 2012. Investigating the performance of neural network backpropagation algorithms for TEC estimations using South African GPS data. 857-866. doi.org/10.5194/angeo-30857-2012.

Haykin, S., 1999. Neural Neural Network and Its Application in IR, a comprehensive foundation, Upper Saddle Rever. New Jersey: Prentice Hall, 842p, 13, 775-781.

Hopfield, J.J., 1982. Neural networks and physical systems with emergent collective computational abilities. Proc. Natl. Acad. Sci. 79 (8), 2554 2558.

Huang, X., Reinisch, B.W., 2001. Vertical electron content from ionograms in real time. Radio Sci. 36 (335-342), 15. https://doi.org/ 10.1029/1999RS002409.

Levenberg, K., 1944. A method for the solution of certain non-linear problems in least squares. Q. Appl. Math. 2 (2), 164-168.

Liu, J., Chen, R., Kuusniemi, H., Wang, Z., Zhang, H., Yang, J., 2009. Mapping the regional ionospheric TEC using a spherical cap harmonic model and IGS products in high latitudes and the arctic region. Proceedings of IAIN 2009 World Congress, Stockholm, October 27.

Mao, T., Wan, W.-X., Liu, L.-B., 2005. An EOF based empirical model of TEC over Wuhan. Chin. J. Geophys. 48 (4), 827-834.

Mao, T., Wan, W., Yue, X., Sun, L., Zhao, B., Guo, J., 2008. An empirical orthogonal function model of total electron content over China. Radio Sci. 43, RS2009. https://doi.org/10.1029/2007RS003629.

Marquardt, D.W., 1963. An algorithm for least-squares estimation of nonlinear parameters. J. Soc. Ind. Appl. Math. 11 (2), 431-441.

Matamba, T.M., Habarulema, J.B., McKinnell, L.A., 2015. Statistical analysis of the ionospheric response during geomagnetic storm conditions over South Africa using ionosonde and GPS data. Space Weather 13 (9), 536-547.

Qahwaji, R., Colak, T., 2006. Neural network-based prediction of solar activities. CITSA2006: Orlando, 4-7.

Okoh, D., 2016. Computer Neural Networks on MATLAB. Createspace, North Charleston, SC, USA, ISBN-13: 978-1539360957.

Okoh, D., Seemala, G., Rabiu, B., Habarulema, J.B., 2019. A neural network - based ionospheric model over africa from constellation observing system for meteorology, ionosphere, and climate and ground global positioning system observations journal of geophysical research: space. Physics. 1-21. https://doi.org/10.1029/2019JA027065.

Pignalberi, A., Pezzopane, M., Rizzi, R., 2018. Modeling the lower part of the topside ionospheric vertical electron density profile over the European region by means of Swarm satellites data and IRI UP method. Space Weather 16, 304-320. https://doi.org/10.1002/ 2017SW001790.

Powers, D.M.W., 2011. Evaluation: From Precision, Recall and FMeasure to Roc, Informedness, Markedness and Correlation. Machine Learning Technology 2(1), 37-63.

Prölss, G., 1993a. Common origin of positive ionospheric storms at middle latitudes and the geomagnetic activity effect at low latitudes. J. Geophys. Res.: Space Phys. 98 (A4), 5981-5991.

Prölss, G., 1995. Ionospheric F-region storms. Handbook Atmos. Electrodyn. 2, 195-248.

Ren, X., Chen, J., Zhang, X., Yang, P., 2020. Topside Ionosphere of NeQuick2 and IRI-2016 validated by using onboard GPS observations from multiple LEO satellites. J. Geophys. Res.: Space Phys. 125 (9), e2020JA027999.

Song, R., Zhang, X., Zhou, C., Liu, J., He, J., 2018. Predicting TEC in China based on the neural networks optimized by genetic algorithm. Adv. Space Res. 62 (4), 745-759. https://doi.org/10.1016/j. asr.2018.03.043.

Stankov, S., Stegen, K., Warnant, R., 2010. Seasonal variations of stormtime TEC at European middle latitudes. Adv. Space Res. 46 (10), $1318-1325$.

Swarm, 2013, https://earth.esa.int/documents/10174/1514862/Swarm_ Level2_TEC_Product_Description.

Stehman, S.V., 1997. Selecting and interpreting measures of thematic classification accuracy. Remote Sensing of Environment 62(1), 77-89. https://doi.org/10.1016/s0034-4257(97)00083-7. 
Tariku, Y.A., 2020. Pattern of the variation of the TEC extracted from the GPS, IRI 2016, IRI-Plas 2017 and NeQuick 2 over polar region, Antarctica. Life Sci. Space Res. 25, 18-27.

Tsagouri, I., Belehaki, A., Moraitis, G., Mavromichalaki, H., 2000. Positive and negative ionospheric disturbances at middle latitudes during geomagnetic storms. Geophys. Res. Lett. 27 (21), 3579-3582.

Tulunay, E., Senalp, E.T., Radicella, S.M., 2006. Forecasting total electron content maps by neural network technique. 41, 1-12. doi.org/ $10.1029 / 2005$ RS003285.

Uwamahoro, J.C., Habarulema, J.B., 2015. Modelling total electron content during geomagnetic storm conditions using empirical orthogonal functions and neural networks. J. Geophys. Res. Space Phys. 120 (12), 11000-11012. https://doi.org/10.1002/2015JA021961.
Watthanasangmechai, K., Supnithi, P., Lerkvaranyu, S., Tsugawa, T., Nagatsuma, T., Maruyama, T., 2012. TEC prediction with neural network for equatorial latitude station in Thailand. Earth Planets Space 64 (6), 473-483.

Wintoft, P., Cander, L.R., 2000. Ionospheric fof 2 storm forecasting using neural networks. Phys. Chem. Earth C Sol. Terr. Planet Sci. 25, 267273. https://doi.org/10.1016/S1464-1917(00)00015-5.

Xiong, C., Stolle, C., Lühr, H., 2016b. The Swarm satellite loss of GPS signal and its relation to ionospheric plasma irregularities. Space Weather 14, 563-577. https://doi.org/10.1002/2016SW001439.

Zakharenkova, I., Astafyeva, E., 2015. Topside ionospheric irregularities as seen from multisatellite observations. J. Geophys. Res. Sp. Phys. 120 (1), 807-824. https://doi.org/10.1002/2014JA020330. 